OPEN ACCESS

Edited by:

Julien Valette,

Commissariat à l'Energie Atomique et aux Energies Alternatives (CEA),

France

Reviewed by:

Valerij G. Kiselev,

Universitätsklinikum Freiburg,

Germany

Silvia Capuani,

Consiglio Nazionale Delle Ricerche

(CNR), Italy

*Correspondence:

Ileana O. Jelescu

ileana.jelescu@epfl.ch

Matthew D. Budde

mdbudde@mcw.edu

Specialty section:

This article was submitted to

Biomedical Physics,

a section of the journal

Frontiers in Physics

Received: 28 September 2017 Accepted: 10 November 2017 Published: 28 November 2017

Citation:

Jelescu IO and Budde MD (2017)

Design and Validation of Diffusion MRI Models of White Matter

Front. Phys. 5:61

doi: 10.3389/fphy.2017.00061

\section{Design and Validation of Diffusion MRI Models of White Matter}

\author{
Ileana O. Jelescu ${ }^{1 *}$ and Matthew D. Budde ${ }^{2 *}$ \\ ${ }^{1}$ Centre d'Imagerie Biomédicale, École Polytechnique Fédérale de Lausanne, Lausanne, Switzerland, ${ }^{2}$ Department of \\ Neurosurgery, Zablocki VA Medical Center, Medical College Wisconsin, Milwaukee, WI, United States
}

Diffusion MRI is arguably the method of choice for characterizing white matter microstructure in vivo. Over the typical duration of diffusion encoding, the displacement of water molecules is conveniently on a length scale similar to that of the underlying cellular structures. Moreover, water molecules in white matter are largely compartmentalized which enables biologically-inspired compartmental diffusion models to characterize and quantify the true biological microstructure. A plethora of white matter models have been proposed. However, overparameterization and mathematical fitting complications encourage the introduction of simplifying assumptions that vary between different approaches. These choices impact the quantitative estimation of model parameters with potential detriments to their biological accuracy and promised specificity. First, we review biophysical white matter models in use and recapitulate their underlying assumptions and realms of applicability. Second, we present up-to-date efforts to validate parameters estimated from biophysical models. Simulations and dedicated phantoms are useful in assessing the performance of models when the ground truth is known. However, the biggest challenge remains the validation of the "biological accuracy" of estimated parameters. Complementary techniques such as microscopy of fixed tissue specimens have facilitated direct comparisons of estimates of white matter fiber orientation and densities. However, validation of compartmental diffusivities remains challenging, and complementary MRI-based techniques such as alternative diffusion encodings, compartment-specific contrast agents and metabolites have been used to validate diffusion models. Finally, white matter injury and disease pose additional challenges to modeling, which are also discussed. This review aims to provide an overview of the current state of models and their validation and to stimulate further research in the field to solve the remaining open questions and converge toward consensus.

Keywords: diffusion MRI, white matter, modeling, microstructure, tissue compartments

Diffusion weighted MRI (DWI) is unique in its ability to detect brain microstructure non-invasively. Characterization of white matter microstructure using DWI has shown high sensitivity to changes associated with normal brain development and aging and to the wide array of neurological diseases, injuries, and potential treatments. DWI is an excellent probe of microstructure, whose characteristic features are on the same length scale as the micrometer-scale displacement of water molecules. However, it remains an indirect probe, because extracting quantitative metrics that characterize the underlying tissue microstructure requires modeling of the DWI signal. Decisions regarding model choice and fitting procedures have considerable influence on the accuracy, reliability, and validity of the extracted metrics. 
This review first presents an overview of current white matter models "in circulation" and exposes their common features and individual limitations (Part 1), noting that the discussion is restricted to long-time limit diffusion models, where axons are modeled as sticks. Next, efforts to validate models in the normal white matter using complementary techniques are discussed (Part 2), followed by a brief discussion of the additional challenges related to modeling of the diseased tissue (Part 3). Future directions of research are highlighted at every step.

\section{MODELS}

Two complementary approaches have emerged for extracting information about the tissue microstructure from the diffusion signal: signal representations and tissue models.

Signal representations-sometimes also referred to as "statistical models"-aim at empirically describing the diffusion signal behavior in a given voxel without assumptions about the underlying tissue. Thus, they are applicable to any tissue type, healthy or diseased, but the estimated parameters lack specificity and remain an indirect characterization of microstructure.

Tissue models, on the other hand, assume a given (schematic) geometry-a "picture" of the underlying tissue [1]. The analytical expression of the diffusion signal in the chosen environment is fit to the diffusion data, which allows the estimation of the relevant parameters of the microstructure. Tissue models can potentially provide greater specificity and interpretation of biologicallyrelevant parameters, but only if the assumption is met that the chosen model accurately captures all of the relevant features of the tissue, i.e., all those that effectively and substantially impact the diffusion signal in a given acquisition range.

In this section, we will first briefly review examples of signal representations, in order to reinforce the distinction with modeling. We will then review major biophysical models of white matter, with clear identification of their main assumptions and estimated parameters.

\section{Signal Representations}

The most widespread signal representation is the cumulant expansion [2-4], which relies on an expansion of the logarithm of the signal in polynomials up to a given order in $b$-i.e., a Taylor series:

$$
\ln \left(\frac{S}{S_{0}}\right)=-b D+\frac{1}{6}(b D)^{2} K+\ldots
$$

where $D$ is the diffusion coefficient and $K$ the kurtosis. Or, in tensor form:

$$
\ln \left(\frac{S}{S_{0}}\right)=-b \sum_{i, j=1}^{3} g_{i} g_{j} D_{i j}+\frac{1}{6}(b \bar{D})^{2} \sum_{i, j, k, l=1}^{3} g_{i} g_{j} g_{k} g_{l} W_{i j k l}+\ldots
$$

where $D$ is the rank-2 diffusion tensor, $W$ is the rank-4 kurtosis tensor, $\bar{D}$ is the mean diffusivity and $\boldsymbol{g}$ is the direction of the applied diffusion weighting.

Diffusion tensor imaging (DTI) is thus an expansion up to the first order in $b$, valid for low diffusion weighting $(b \ll 1 /(D K))$
[5]. It is important to stress that DTI does not assume that the tissue is a Gaussian medium $(K=0)$ - which is reputedly not true for biological tissue, but rather that it is indistinguishable from a Gaussian medium when $b \ll 1 /(D K)$.

Naturally, going beyond this low $b$-value regime and estimating the kurtosis of the diffusion probability distribution function-i.e., by how much it deviates from a Gaussian distribution-provides information about tissue complexity that is complementary to DTI metrics [2].

In order to estimate the six independent components of the diffusion tensor, the minimal required data is one $b=0$ (unweighted) image and six non collinear directions on a single diffusion weighting, or "shell." The additional estimation of the 15 independent components of the kurtosis tensor requires a minimal acquisition of one $b=0$ image and 21 measurements distributed over 2 shells. The choice of the shell $b$-values is a trade-off between accuracy-they should be as low as possible, since the Taylor expansion is valid for $b D \rightarrow 0-$ and precisionthey should be as high as admittedly possible to limit the impact of noise [6]. This trade-off value is typically around $b=1$ $\mathrm{ms} / \mu \mathrm{m}^{2}$ for DTI and $b=2 \mathrm{~ms} / \mu \mathrm{m}^{2}$ for DKI, in vivo. Largely improved algorithms for unbiased estimation of the diffusion and kurtosis tensors have been developed [7]. It is also noteworthy that fitting the kurtosis tensor greatly improves the accuracy of the diffusion tensor estimation [8]. Extending the series to the sixth order cumulant (in $b^{3}$ ) increases the accuracy of the kurtosis estimation, albeit with a penalty on precision [9].

Since no assumption is made about the tissue structure, metrics derived from the diffusion tensor, such as mean diffusivity and fractional anisotropy are used extensively for brain and body tissue characterization in a wide variety of conditions $[10,11]$. DKI metrics, such as mean kurtosis, are also increasingly used as complementary biomarkers to DTI metrics in a large panel of applications [12-17]. With acquisition acceleration options available on most clinical scanners, and new reduced acquisition schemes $[18,19]$, DKI is expected to become more and more widespread.

Other signal representations than the cumulant expansion are of course possible. Yablonskiy et al. [20] proposed to introduce a distribution of diffusion coefficients $\rho(D)$ in the tissue, which can theoretically be estimated by inverse Laplace transform:

$$
\frac{S}{S_{0}}=\int_{0}^{\infty} \rho(D) e^{-b D} d D
$$

However, because the inverse Laplace transform is a mathematically ill-posed problem, in practice some functional form is assumed for $\rho(D)$ and its characteristic parameters estimated [20, 21]. Moreover, the estimated distribution does not mirror the actual distribution of diffusion coefficients in the tissue unless the measurement is performed in a very strong diffusion weighting regime $\left(q l_{c} \gg 1\right.$, where $q$ is the amount of spatial phase-warping introduced and $l_{\mathrm{c}}$ is the diffusion distance) $[9,22]$. Thus, this approach remains an empirical description of 
the diffusion-weighted signal and falls in the category of signal representations rather than biophysical models.

The departure from Gaussianity can also be captured empirically as a "stretched exponential" expression of the diffusion signal [23]:

$$
\frac{S}{S_{0}}=\exp \left(-(b \cdot \mathrm{ADC})^{\alpha}\right)
$$

The stretched exponential function has later been associated with the theory of anomalous diffusion, which represents a departure from Brownian motion $\left(\left\langle x^{2}\right\rangle \propto t\right)$, by several groups [24-26]. A comprehensive discussion on the implications of such a theory for biological tissue can be found in Kiselev [9] and Novikov et al. [1]. Although devoid of biophysical meaning, the stretching parameter $\alpha$ is largely used in the literature for tumor delineation and characterization [27-30].

Another example of signal representation is the expansion of the diffusion signal using harmonic oscillator basis functions (Hermite polynomials), as proposed by Özarslan et al. [31], a method dubbed mean apparent propagator (MAP-MRI). In addition to the reconstruction of a diffusion tensor, the method allows the estimation of the return-to-origin probability, which is sensitive to compartment sizes, and of non-Gaussianity indices, indicative of tissue complexity. The data required for MAP-MRI can be acquired in under $10 \mathrm{~min}$ on a clinical scanner [32].

\section{Biophysical Models of White Matter}

The first attempts at modeling the diffusion signal as multicompartmental can be attributed to Latour et al. [33] in red blood cells and Szafer et al. [34] who modeled "tissue" as cuboids on a regular lattice, surrounded by extracellular medium. Building on their work, Stanisz et al. [35] proposed the first white matter model based on electron micrographs of bovine optic nerve. In their model, axons were represented as prolate ellipsoids and glial soma as spheres, the extracellular space constituting the third compartment. The model also accounted for membrane permeability of axons and glial cells. The authors noted that the nine-parameter model required a wide range of diffusion times and gradient strengths, and that even with extensive data, the goodness of fit remained unsatisfactory. They also underlined that releasing some of the assumptions and increasing the number of parameters would make the fit unstable. Twenty years later, those insights are still very much topical.

At long diffusion times, the diffusion distance across the axon becomes constant (it is limited by the axon diameter) and the diffusivity tends to zero: axons can thus be modeled as infinitely long "sticks" (cylinders with zero radius). The first group to introduce the stick geometry was Behrens et al. [36] and assumed that water in and around the axons similarly diffused only in the fiber direction with a second compartment of free, isotropically diffusing water. Shortly after, Kroenke et al. [37] used a biophysical model of isotropically oriented sticks to characterize the diffusion of N-acetyl aspartate (NAA) in the neurites. A similar description to Behrens' of two compartments was later adopted by Jespersen et al. [38], who also formulated for the first time the clear goal of estimating dendrite density-i.e., a specific biomarker of the microstructure-in vivo, using diffusion MRI modeling.

Building on these initial works, most white matter models "in use" today rely on the same common picture: water signal is assumed to originate from two or three non-exchanging compartments, each weighted by their relative volume fractions (Figure 1).

The first compartment is the collection of axons, which are modeled as infinitely long sticks, and whose collective orientations are characterized by some orientation distribution function (ODF). Water diffusivity inside each axon is $D_{\mathrm{a}, \|}$ along the axon and zero perpendicular to it. The parametrization of the ODF varies between models.

The second compartment is the extra-axonal space which, by exclusion, includes features not explicitly ascribed to the axonal space, including extracellular water, cell somas, and glial cells, all assumed to be in fast exchange. This compartment is modeled as Gaussian anisotropic, with axial and radial diffusivities $D_{\mathrm{e}, \|}$
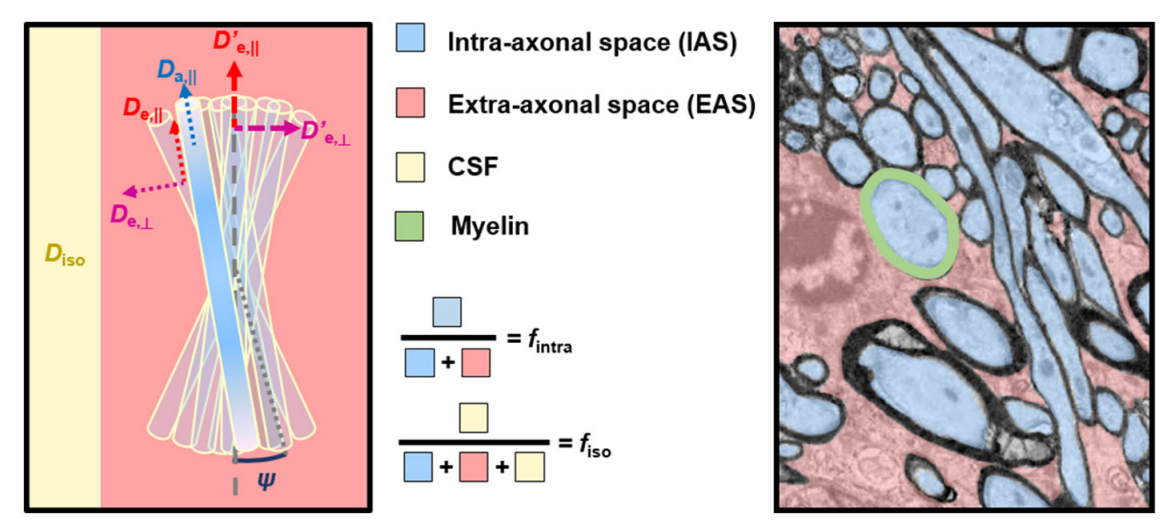

FIGURE 1 | Correspondence between model compartments (Left) and tissue components (Right). (Left) Schematic of a typical three-compartment model with relevant parameters. $D_{e, \|}$ and $D_{e, \perp}$ are local extra-axonal diffusivities, and $D_{e, \|}^{\prime}$ and $D_{e, \perp}^{\prime}$, are apparent extra-axonal diffusivities, depending how the model defines them. (Right) Cross-sectional electron microscopy image of a white matter bundle. Adapted from Mikula and Denk [39] with permission. While myelin is present in WM, it is absent from DWI models due to its short $T_{2}$. 
and $D_{e, \perp}$, respectively. These extra-axonal diffusivities are either defined locally in proximity of a coherent axon sub-bundle with the resulting signal response convolved with the ODF, or as apparent extra-axonal diffusivities over the entire voxel (indicated with a "prime" symbol in Figure 1 and hereafter, for disambiguation).

The third compartment, if included, is modeled as Gaussian isotropic, with a diffusivity $D_{\text {iso. If it represents freely diffusing }}$ water such as cerebrospinal fluid (CSF), $D_{\text {iso }}\left(D_{\text {free }}\right)$ is set to $3 \mu \mathrm{m}^{2} / \mathrm{ms}$ in vivo. If it represents water with a negligible diffusivity in all directions, often referred to as "still water," and non-exchanging with the extracellular space, $D_{\text {iso }}=0$. The parametrization, constraints, and nomenclature of $D_{\text {iso }}$ vary between models.

Modeling necessarily comes with a level of simplification, since the entire biological complexity certainly cannot be rendered. The most difficult part of the modeling task is perhaps figuring out which features of the microstructure are relevant to the voxel-averaged diffusion signal and should be represented in the model, and which ones can be omitted without violating assumptions or accuracy. For a more detailed discussion on coarse-graining and effective medium theory, we direct the reader to a very comprehensive review by Novikov et al. [1].

In what follows, we will briefly review the main white matter models that have, for the most part, emerged in the last decade and that share the common features outlined above. While many different diffusion models and corresponding applications have been proposed, this review will only focus on models of tissue microstructure pertaining to the brain and spinal cord and in the long-time limit where axons are modeled as sticks. We also neglect potential measures of permeability between compartments, though they could have substantial impact on modeling, especially in the case of pathologies [40].

\section{NODDI}

The tissue model in Neurite Orientation Dispersion and Density Imaging (NODDI) matches exactly the cartoon in Figure $\mathbf{1}$ [41]. It is a three-compartment model fully described by seven parameters: two volume fractions $\left(f_{\text {intra }}\right.$ and $\left.f_{\text {iso }}\right)$, four diffusivities $\left(D_{\mathrm{a}, \|}, D_{\mathrm{e}, \|}, D_{\mathrm{e}, \perp}\right.$, and $\left.D_{\mathrm{iso}}\right)$ and the orientation dispersion, modeled by a Watson distribution of concentration parameter $\kappa$. The latter can be related to the angular spread via

$$
\left\langle(\cos \psi)^{2}\right\rangle=-\frac{1}{2 \kappa}+\frac{1}{\sqrt{\pi} e^{-\kappa} \operatorname{erfi}(\sqrt{\kappa}) \sqrt{\kappa}} .
$$

where $\psi$ is the angle between an axon and the main diffusion orientation in the voxel. In order to stabilize the fitting procedure, NODDI constrains all diffusivity values, effectively leaving only the two volume fractions and the orientation dispersion to be estimated, i.e., the underlying geometry. The model assumptions are as follows:

$$
\begin{array}{r}
D_{a, \|}=D_{e, \|}=1.7 \mu \mathrm{m}^{2} / \mathrm{ms} \\
D_{e, \perp}=\left(1-f_{\text {intra }}\right) \cdot D_{e, \|} \\
D_{i s o}=3 \mu \mathrm{m}^{2} / \mathrm{ms}
\end{array}
$$

The popularity of NODDI is indisputable, with applications to a very large panel of brain alterations and pathologies [4248]. However, there are important assumptions within NODDI related to its design that have strong implications to its specificity.

NODDI imposes assumptions on the intrinsic diffusivities to the point of fixing $D_{\mathrm{a}, \|}$ and $D_{\mathrm{e}, \|}$ to the same predetermined value for all tissues (Equation 5). First, any deviation from these fixed values can bias the remaining parameters and they will lose their desired specificity. Substantial changes in diffusivity, such as that occurring in cerebral ischemia, for example, will impart a false change in orientation dispersion, neurite density, or CSF partial volume that does not accurately capture the underlying tissue pathology - as will be discussed in more detail in the third part of this review. The decision to fix the axial intra- and extraaxonal diffusivities equal to one another is another assumption that can lead to unpredictable effects. Recent work has shown that fixing $D_{\mathrm{a}, \|}=D_{\mathrm{e}, \|}$ masks a fundamental property of multicompartment models, namely the multiplicity of mathematical solutions [49,50]. Indeed, it has been shown that, if all parameters in the fitting procedure are released, namely $f_{\text {intra }}, D_{\mathrm{a}, \|}, D_{\mathrm{e}, \|}$, $D_{\mathrm{e}, \perp}$, and $\kappa$ (ignoring the CSF compartment), there are two distinct solutions to the parameter estimation problem, both within biologically plausible ranges (Figure 2). The two solutions of a two-compartment model can roughly be described as one where $D_{\mathrm{a}, \|}<D_{\mathrm{e}, \|}$, and another where $D_{\mathrm{a}, \|}>D_{\mathrm{e}, \|}$. Establishing which inequality is biologically valid is an active field of research, with most studies pointing toward $D_{\mathrm{a}, \|}>D_{\mathrm{e}, \|}$. This will be covered in detail in the second part of the review. Finally, the tortuosity approximation that relates $D_{\mathrm{e}, \|}$ and $D_{\mathrm{e}, \perp}$ (Equation 6) has been shown not to hold for tight packings of axons [51].

\section{WMTI}

White Matter Tract Integrity (WMTI) is a two-compartment model of sticks embedded in a Gaussian anisotropic extra-axonal medium [52]. The tissue can be described as a combination of two Gaussian compartments (intra- and extra-axonal) each characterized by a tensor $\left(\hat{D}_{a}\right.$ and $\left.\hat{D}_{e}^{\prime}\right)$ which can be directly derived from the overall diffusion and kurtosis tensors $\hat{D}$ and $\hat{K}$ [53]. In any direction $j$,

$$
\begin{array}{r}
D_{j}=f_{\text {intra }} D_{a, j}+\left(1-f_{\text {intra }}\right) D^{\prime}{ }_{e, j} \\
K_{j}=3 f_{\text {intra }} \cdot\left(1-f_{\text {intra }}\right) \frac{\left(D_{e, j}^{\prime}-D_{a, j}\right)^{2}}{D_{j}^{2}}
\end{array}
$$

This approach by-passes inherent limitations associated with non-linear fitting (local minima and long computation time): the linear estimation of the diffusion and kurtosis tensors is followed by a direct derivation of the WMTI model parameters. The quadratic expression in Equation (9) demonstrates, as previously mentioned, that for a two-compartment model, there are two mathematical solutions, where either $D_{\mathrm{a}, \|}<D_{\mathrm{e}, \|}$, or $D_{\mathrm{a}, \|}>D_{\mathrm{e}, \|}$. Establishing which inequality is biologically valid is an active field of research, which will be covered in detail in the second part of the review.

In WMTI, the authors had chosen the inequality $D_{\mathrm{a}, \|}<D_{\mathrm{e}, \|}$ to solve the system (which is the opposite of the inequality that 

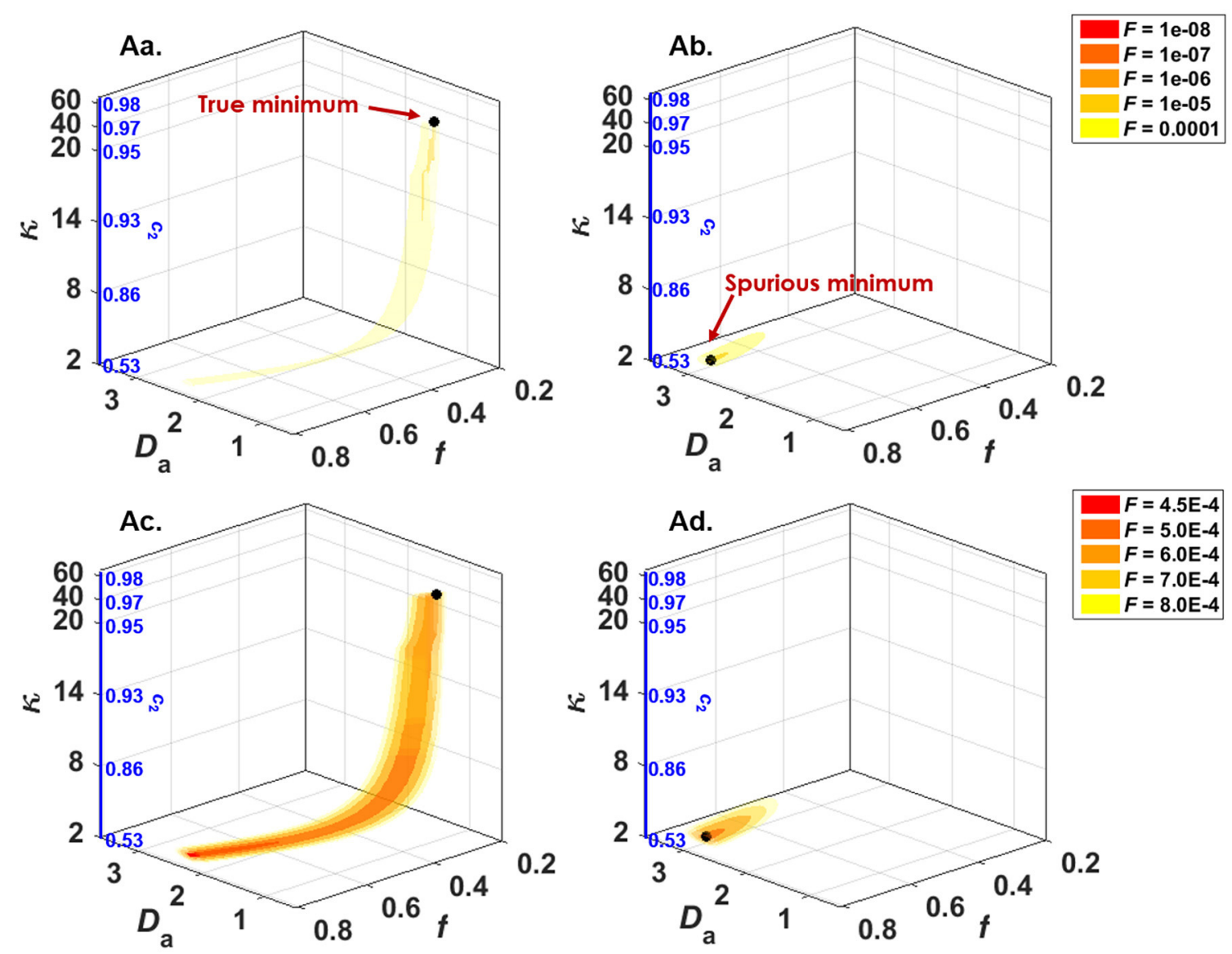

$F=4.5 \mathrm{E}-4$ $F=5.0 \mathrm{E}-4$ $F=6.0 \mathrm{E}-4$ $F=7.0 \mathrm{E}-4$ $F=8.0 \mathrm{E}-4$

FIGURE 2 | NODDIDA optimization landscape reveals the existence, in the full 5-parameter space, of two disconnected minima, each surrounded by a pipe-like ensemble of low objective function values. Shown are 3D isosurfaces of the objective function $F\left(f, D_{\mathrm{a}, \|}, \kappa\right)$. (Aa) $F$ calculated with $\left(D_{\mathrm{e}, \|} ; D_{\mathrm{e}, \perp}\right)=(2.10 ; 0.74)$ and $\mathrm{SNR}$ $=\infty$, thus containing the true global minimum. (Ab) $F$ calculated with $\left(D_{e, \|} ; D_{e, \perp}\right)=(0.32 ; 0.85)$ and $S N R=\infty$, thus containing the second local minimum of the $5 D$ minimization problem. (Ac) The same as (Aa), but SNR $=50$. (Ad) The same as (Ab), but SNR $=50$. The theoretical minimum of $F$ along each pipe is identified by a black bullet. Noise can displace the effective minimum along the pipe very far from the true one (see Ac). Figure taken from Jelescu et al. [49], with permission.

seems to hold from recent work), hence:

$$
\begin{array}{r}
f_{\text {intra }}=\frac{K_{\max }}{K_{\max }+3} \\
D^{\prime}{ }_{\mathrm{e}, \mathrm{j}}=D_{\mathrm{j}}\left[1+\sqrt{\frac{K_{j} \cdot f_{\text {intra }}}{3\left(1-f_{\text {intra }}\right)}}\right] \\
D_{\mathrm{a}, \mathrm{j}}=D_{\mathrm{j}}\left[1-\sqrt{\frac{K_{\mathrm{j}}\left(1-f_{\text {intra }}\right)}{3 f_{\text {intra }}}}\right]
\end{array}
$$

WMTI has been used in studies of a large variety of conditions, with substantial validation efforts using animal models of altered myelin [12, 13, 54-58].

In WMTI, the collection of axons is modeled as a Gaussian compartment, which is an assumption that only holds if the axons are highly aligned in a single bundle. Fieremans and colleagues estimated a maximum orientation dispersion of $30^{\circ}$ for this approximation to hold, in the case of coplanar dispersion only. The validity of the approximation for three-dimensional dispersion has not been evaluated. Thus, while WMTI captures changes in diffusivities, it is best applied to regions of highly aligned single fiber bundles as per the recommendations. A more recent approach consists in deriving the WMTI equations assuming a Watson distribution of axons, like in NODDI, thus alleviating one of the strongest assumptions of WMTI [59]. The authors then further use time dependent functional forms of model parameters to evaluate which of the two possible solutions is the biologically valid one.

Another limitation is that since WMTI is based on the kurtosis model, it is restricted to the low $b$-value regime which could lead to some bias, although this may also be advantageous for compatibility with clinical hardware systems.

\section{Rotationally Invariant Features: LEMONADE \& co.}

In a two-compartment model, estimating compartment diffusivities and orientation dispersion of axons simultaneously is problematic and prone to bias, hence requiring fixed parameter assumptions (NODDI) or limitations to coherent fibers only (WMTI). Loosening these constraints to achieve 
greater specificity comes at the expense of a larger number of parameters to be estimated in the model, which is problematic for non-linear fitting. In particular, allowing for a complex ODF quickly increases the number of parameters. NODDI for example also has a declination that uses a Bingham rather than Watson distribution (2 free parameters) [60]. Jespersen and colleagues modeled the ODF using a spherical harmonic expansion up to $L=2$ (5 free parameters) [38] and later $L=4$ (14 free parameters) [61].

For the purpose of extracting a reliable ODF for tractography, several groups had modeled the diffusion signal as a convolution between the response of a perfectly aligned fascicle (axons and their immediate extra-axonal space) pointing in direction $\mathbf{n}$, and the fiber ODF $P(\mathbf{n})[62-64]$ :

$$
S_{\mathbf{g}}(b)=S(0) \cdot \int_{|\mathbf{n}|=1} \mathcal{K}(b, \mathbf{g} \cdot \mathbf{n}) \mathcal{P}(\mathbf{n}) d \mathbf{n}
$$

Equation 13 factorizes in the spherical harmonics basis, thus separating the estimation of the scalar parameters of the kernel $K$ from that of the ODF parameters. Recently, Novikov et al. [50] and Reisert et al. [65] have exploited this property to estimate the scalar parameters of a two-compartment kernel separately from the ODF:

$$
\begin{aligned}
\mathcal{K}(b, \mathbf{g} \cdot \mathbf{n})= & f_{\text {intra }} e^{-b D_{a, \|}(\mathbf{g} \cdot \mathbf{n})^{2}} \\
& +\left(1-f_{\text {intra }}\right) e^{-b D_{e, \|}(\mathbf{g} \cdot \mathbf{n})^{2}-b D_{e, \perp}\left(1-(\mathbf{g} \cdot \mathbf{n})^{2}\right)}
\end{aligned}
$$

The approach of Novikov et al. [50] can be decomposed into two steps. The first step relies on solving an algebraic system of equations which relates the kernel parameters to the signal moments. More specifically, using "low $b$-value" data (e.g., $b_{\max }=2.5 \mathrm{~ms} / \mu \mathrm{m}^{2}$ ), the scalar parameters and a few basisindependent ODF parameters $p_{1}$ can be directly derived from the first moments of the diffusion signal (up to 6th order) in a similar fashion to which WMTI uses direct relationships between model parameters and cumulants up to the 4th order. This method was dubbed LEMONADE (Linearly Estimated Moments provide Orientations of Neurites And their Diffusivities Exactly), and requires diffusion data distributed over 3 non-zero $b$ shells. Circumventing issues related to non-linear fitting, LEMONADE provides estimates for $f_{\text {intra }}, D_{\mathrm{a}, \|}, D_{\mathrm{e}, \|}, D_{\mathrm{e}, \perp}$, and $p_{2}=$ $\frac{3\left|(\cos \psi)^{2}\right\rangle-1}{2}$, which gives an estimate of the orientation dispersion in a similar way to $\kappa$ from the Watson distribution. The spherical harmonic expansion of the ODF can then be fully reconstructed up to $L=6$. In a second step, all available data (including high $b$-values) can be exploited to minimize the rotationally invariant (RotInv) energy function of the system, using the LEMONADE estimates as initialization values. In other words, the projections of the scalar kernel onto Legendre polynomials, weighted by rotational invariants of the ODF, are fitted to rotational invariants of the spherical harmonics decomposition of the signal, in a least-squares sense.

LEMONADE provides further evidence for the existence of two mathematical solutions to the two-compartment model and that, in principle, the degeneracy can be lifted with robust measurements up the $3 \mathrm{rd}$ order in $b$. In practice, solution selection based on noisy data remains challenging and should be validated independently.

The approach of Reisert et al. [65] relies on machine learning to estimate the model parameters. A choice of plausible parameter values is made using insight from isotropic diffusion weighting results, as will be discussed in Validating Microstructural Features. This method also allows renouncing to the determination of specific parameters of the model when data proves insufficient.

The remaining assumptions behind these two methods are: the existence of only two compartments (intra- and extraaxonal), the consistency of kernel parameters across all fascicles in the voxel (e.g., all axons in the voxel have the same diffusivity $\left.D_{\mathrm{a}, \|}\right)$, and axial symmetry of the elementary fiber response (kernel).

By contrast to these two approaches, the method described by Kaden et al. [66] based on the spherical mean technique is similar in spirit, in the sense that it factors out the fiber ODF, but it only exploits the lowest order rotational invariant and the estimation of the scalar kernel is constrained by $D_{\mathrm{a}, \|}=D_{\mathrm{e}, \|}$ and by the tortuosity approximation for the extra-axonal space (Equation 6).

\section{DBSI}

Another model was proposed with the intent of covering regions of multiple fiber crossings; this model is DBSI (Diffusion Basis Spectrum Imaging) [67], and its general formulation is the following:

$$
\begin{aligned}
S_{\mathbf{g}}(b)= & \sum_{i=1}^{N_{\text {Aniso }}} f_{i} \cdot e^{-b D_{\|, \mathbf{i}}\left(\mathbf{g} \cdot \mathbf{n}_{\mathbf{i}}\right)^{2}-b D_{\perp, \mathrm{i}}\left(1-\left(\mathbf{g} \cdot \mathbf{n}_{\mathbf{i}}\right)^{2}\right)} \\
& +\int_{\alpha}^{\beta} f(D) e^{-b D} d D
\end{aligned}
$$

where the tissue is described as a collection of $N_{\text {Aniso }}$ axially symmetric anisotropic tensors (each with unknown fraction $f_{\mathrm{i}}$, axial and radial diffusivities $D_{\|, \mathrm{i}}$ and $D_{\perp, \mathrm{i}}$ and orientation $\mathbf{n}_{\mathrm{i}}$ ) and a continuous spectrum $f(D)$ of isotropic diffusion tensors. The anisotropic tensors represent water inside and just outside myelinated and unmyelinated axons of varied directions, while the continuum of isotropic tensors represents water in cells, sub-cellular structures, and edematous water.

The DBSI fitting procedure is complex and details can be found in Wang et al. [67]. Briefly, it is a two-step process where first the number of anisotropic tensors and their principal orientations are estimated. These parameters are then fixed in the second step of the procedure, which aims at estimating $f_{\mathrm{i}}, D_{\|, \mathrm{i}}$, and $D_{\perp, \mathrm{i}}$ for each anisotropic tensor, as well as $f(\mathrm{D})$. This is achieved by optimizing $f_{\mathrm{i}}$ and $f(\mathrm{D})$ for recursively chosen combinations of $D_{\|, \mathrm{i}}$ and $D_{\perp, \mathrm{i}}$ and finally selecting the combination providing a global minimum.

DBSI has been applied to a variety of pathologies [68-72], but has not been extensively validated outside of the originating group. Given the unfavorable fitting landscape in a comparatively much simpler model such as NODDIDA [49], especially in the presence of limited signal-to-noise ratio (SNR) (Figure 2), the reliability of DBSI model estimates remains to be clarified. 
Moreover, the biophysical interpretation of the continuous distribution of diffusion coefficients $f(D)$ is also problematic, as explained in connection with Equation (3).

\section{Hybrid Models}

Hybrid models are based on a combination of biophysical modeling and signal representations.

\section{Free Water Elimination (FWE)}

The motivation of the free water elimination technique is to separate CSF contamination from "brain tissue" diffusion properties. The overall signal is separated into an isotropic component of free diffusion $\left(D_{\text {free }}\right)$ and a single diffusion tensor representing the rest of the tissue signal $\left(D_{\text {tissue }}\right)$. This approach was first proposed by Pierpaoli and Jones [73] using a comprehensive $q$-space acquisition (107 directions over 8 shells, $b_{\max }=1.2 \mathrm{~ms} / \mu \mathrm{m}^{2}$ ). Its potential advantages are the extraction of a free water map (as a new biomarker), improved tissue tensor estimation, and benefits for fiber tracking. The direct correspondence between "free water" and CSF is however not straightforward.

Its application was further extended to single-shell DTI data by Pasternak et al. [74]. Naturally, estimating a system which includes a tensor and an isotropic compartment from single-shell data is an underdetermined problem. To circumvent this issue, Pasternak and colleagues initially imposed additional constraints: spatial regularization and tighter upper and lower bounds on the tissue volume fraction, dependent on tissue diffusivities. This constrained implementation has been applied to a variety of conditions [75-78].

While the intention of making the method applicable to the most widespread acquisition scheme (i.e., single shell) is understandable, the ill-posed nature of the mathematical problem and the issue of constraints has been recognized by the authors, who proposed incorporation of an additional low $b$-value shell for accuracy and stability [79]. The initial, more conventional, multi-shell approach proposed by Pierpaoli and Jones has also been reintroduced in parallel by Hoy et al. [80]. The latter work included simulations showing that in the absence of a CSF compartment, fitting the FWE model indeed overestimated tissue FA. Later work by the same group compared the FWE technique to the more standard FLAIR-DTI approach where the CSF signal was directly suppressed at the acquisition stage [81, 82]. They reported similar performance between the two techniques in WM tracts where CSF contamination was expected to be high (i.e., fornix and corpus callosum), but unlike FLAIRDTI, the FWE technique resulted in greater FA and reduced diffusivity measures even in tissues without CSF partial volume effects (e.g., cingulum). The proposed explanation that the "free water" signal arises from the extracellular space [79] highlights the complications of assigning a single tensor to a "tissue" compartment which is known to exhibit non-Gaussian behavior.

\section{DIAMOND}

A new hybrid model, termed DIAMOND (DIstribution of 3D Anisotropic MicrOstructural eNvironments in Diffusioncompartment imaging) [83] has recently been proposed. It expands on the concept of distribution of diffusion coefficients introduced by Yablonskiy et al. [20], by accounting for several discrete compartments (biophysical modeling), each withholding a continuous distribution of diffusion tensors (statistical modeling). In mathematical terms:

$$
S_{\mathbf{g}}(b)=S_{0} \sum_{i=1}^{N_{p}} f_{i} \cdot \int_{\mathbf{D} \in S_{y m}+(3)} P_{\kappa_{i}, \Sigma_{i}}(\mathbf{D}) e^{-b \mathbf{g}^{T} \mathbf{D g}} d \mathbf{D}
$$

where $N_{\mathrm{p}}$ is the number of compartments (or spin populations), each with its associated volume fraction, and $P_{\kappa_{i}, \Sigma_{i}}(\mathbf{D})$ is the distribution of diffusion tensors within the compartment, chosen to be a multi-variate Gamma distribution of shape parameter $\kappa$ and scale parameter $\Sigma$. The diffusion tensors are symmetric positive definite matrices $\left[\operatorname{Sym}^{+}(3)\right]$.

Starting from the generic expression in Equation (16), three types of particular compartments are considered and their presence in each voxel is evaluated using model selection techniques. The three types of compartments are free isotropic (attributed to CSF), restricted isotropic (attributed to water in glial cells) and water in and around white matter fascicles.

In practice, DIAMOND uses an iterative approach in defining the number of compartments in each voxel. The optimization begins with a freely-diffusing compartment and zero fascicles, and the number of fascicles is gradually increased up to threethe higher complexity is retained only if the general error of the model is significantly reduced compared to the previous simpler model. Spatial regularization of voxel parameters is also used. While the goodness of fit has been extensively used in the past to choose which model is most appropriate for describing the data $[84,85]$, recent insights into the topology of the fitting landscape for a two-compartment model (Figure 2) advises against relying solely on such metrics for model selection $[49,50]$.

As an extension of the concept of continuous distribution of diffusion coefficients (Equation 3), DIAMOND also inherits from its associated issues, discussed in the section on Signal Representations.

Microstructure metrics estimated using DIAMOND will need to be subjected to substantial validation studies in the near future. Recent work on traumatic brain injury in the mouse suggests that DIAMOND, as any higher order model, is more sensitive than DTI in detecting differences, but a more thorough validation of specificity against histology remains to be performed [86].

\section{Summary}

Main approaches for deriving quantities from the diffusion weighted signal include signal representations (also known as statistical models), biophysical models of white matter, and hybrid models that contain features of both approaches. Table 1 summarizes the properties of each of these approaches. Essentially, single-shell diffusion MRI data only enables the estimation of a single diffusion tensor for the voxel, i.e., DTI. Any other more complex analysis that accounts for non-Gaussian diffusion effects in the tissue requires at least two non-zero $b$-shells, each with sampling along a minimum number of directions.

Biophysical models come with the promise of characterizing tissue microstructure with improved specificity. However, their intrinsic assumptions have the potential to introduce bias. 
TABLE 1 | Summary of method characteristics.

\begin{tabular}{|c|c|c|c|c|}
\hline & Method & Minimum/typical data requirements & Estimated metrics & Assumptions/constraints \\
\hline \multirow[t]{2}{*}{ Statistical models } & DTI & $1 b=0 ; 6 b=1$ & $\begin{array}{l}\text { Diffusion tensor (and derived FA, MD, AD, } \\
\text { RD) }\end{array}$ & None \\
\hline & $\mathrm{DKI}$ & $1 b=0 ; 6 b=1 ; 15 b=2$ & $\begin{array}{l}\text { Same as DTI + kurtosis tensor (and } \\
\text { derived MK, AK, RK) }\end{array}$ & None \\
\hline \multirow[t]{5}{*}{ Biophysical models } & NODDI & $1 b=0 ; 30 b=0.7 ; 60 b=2$ & $f_{\text {intra }}, f_{\text {iso }}, \kappa$ & $\begin{array}{l}D_{\mathrm{a}, \|}=D_{\mathrm{e}, \|}=1.7 \\
D_{\mathrm{e}, \perp}=\left(1-f_{\text {intra }}\right) D_{\mathrm{e}, \|} \\
D_{\text {iso }}=3\end{array}$ \\
\hline & WMTI & $1 b=0 ; 6 b=1 ; 15 b=2$ & $f_{\text {intra }}, D_{\mathrm{a}, \|}, D_{\mathrm{e}, \|}, D_{\mathrm{e}, \perp}$ & $\begin{array}{l}\text { Limited dispersion } \\
f_{\text {iso }}=0 \\
D_{\mathrm{a}, \|} \leq D_{\mathrm{e}, \|}\end{array}$ \\
\hline & $\begin{array}{l}\text { LEMONADE / } \\
\text { RotInv }\end{array}$ & $\begin{array}{l}1 b=0 \text {; minimum } 3 \text { non-zero shells with } \\
\text { e.g., } 64 \text { dirs per shell }\end{array}$ & $\begin{array}{l}f_{\text {intra }}, D_{\mathrm{a}, \|}, D_{\mathrm{e}, \|}, D_{\mathrm{e}, \perp} \\
\mathrm{SH} \text { up to } 6 \text { th order for ODF }\end{array}$ & $f_{\text {iso }}=0$ \\
\hline & $\begin{array}{l}\text { Reisert et al. } \\
\text { [65] }\end{array}$ & $\begin{array}{l}2 \text { non-zero shells or uniform filling of } \\
\text { q-space }\end{array}$ & $f_{\text {intra }}, f_{\text {iso }}, D_{\mathrm{a}, \|}, D_{\mathrm{e}, \|}, D_{\mathrm{e}, \perp}$ & $\begin{array}{l}\text { Some parameters will not be } \\
\text { estimated if insufficient data }\end{array}$ \\
\hline & DBSI & 99 directions over $9 b$-shells, $b_{\max }=1$ & $\begin{array}{l}\text { Number of anisotropic tensors } \mathrm{N} \text { and their } \\
\text { respective } f_{\mathrm{i}}, \lambda_{\|, \mathrm{i}} \text { and } \lambda_{\perp, \mathrm{i}},+ \text { isotropic } \\
\text { tensor distribution } f(\mathrm{D})\end{array}$ & $\begin{array}{l}\text { Two-step fitting process, with some } \\
\text { parameters temporarily fixed }\end{array}$ \\
\hline \multirow[t]{2}{*}{ Hybrid models } & FWE & $1 b=0 ; 32 b=0.5 ; 32 b=1.5$ & $f_{\text {iso }}+$ tissue diffusion tensor & $\begin{array}{l}D_{\text {iso }}=3 \\
\text { Tissue described by a tensor }\end{array}$ \\
\hline & DIAMOND & $\begin{array}{l}\text { "CUSP90": } \\
12 b=0 ; 6 b=0.4 ; 6 b=0.6 ; 6 b=0.8 \\
30 b=1 \\
+30 \text { directions on a cube }(1 \leq b \leq 3)\end{array}$ & $\begin{array}{l}f_{\text {iso, }} \text {, number of fascicles (up to } 3 \text { ) and their } \\
\text { respective } f_{\mathrm{i}}, \kappa_{\mathrm{i}} \text { and } \Sigma_{\mathrm{i}}\end{array}$ & $\begin{array}{l}\text { Iterative fitting for number of } \\
\text { compartments; } \\
\text { Spatial regularization; } \\
\text { mv- } \Gamma \text { distribution of tensors within } \\
\text { compartment }\end{array}$ \\
\hline
\end{tabular}

b-values are in $\mathrm{ms} / \mu \mathrm{m}^{2}$ and diffusivities in $\mu \mathrm{m}^{2} / \mathrm{ms}$.

Nearly all models outperform DTI in capturing tissue diffusion properties, but this is a very low benchmark. Biophysical models need to be increasingly compared to one another, and to higher order signal representations such as DKI, to understand convergent model properties likely to be successful in broad applications [57, 65, 87, 88]. Their reliability in the case of pathological tissue also needs thorough investigation.

\section{VALIDATING DIFFUSION MODELS}

Most biophysical diffusion models share at least partial similarity with one another with respect to their common geometry, as represented in Figure 1. This section will review validation efforts for each of the model features (compartment volume fractions, axon orientation dispersion, and compartment diffusivities). While many validation studies have been performed for DTI, the goal is to emphasize results in the context of biophysical models and how well their derived parameters reflect the true biology of interest. The first two sub-sections focus on method validation using either computer simulations or phantoms, while the following ones discuss the validation of quantitative estimates for each microstructural feature of white matter.

\section{Computational Simulations}

Simulating the random walk of diffusing spins within simple or complex environments is often the first step to establish a connection between derived estimates of physical features and their ground truth values. Simulations have been used where analytic solutions are difficult or impossible, and their use is so common that it is impossible to list them here. Notable examples are those that have also released accompanying software packages [89-92]. Synthetic geometries, which are typically composed of cylindrical axons, have been used to model the effects of axon diameter, packing, volume fractions, permeability, and other features that can be easily modulated synthetically, and these have in turn been performed under many different simulated experimental conditions such as modulation of diffusion times, gradient waveform shapes, signal to noise ratios, and other features that would be difficult or time-consuming to perform experimentally. As the diffusion models have evolved to include more complex features such as bending, crossing, and asymmetric fiber orientations, simulations have followed suit and implemented similar physical features. In addition to purely fiber models, other notable examples include complex axonal geometries to demonstrate the effects of swelling [93], beading [94], undulation [95], and other features. Geometrical substrates derived from tissue specimens [96] have been also demonstrated recently, although these remain challenging due to the difficulties in obtaining high-resolution, 3D images of tissues and their subsequent conversion to physical substrates. As $3 \mathrm{D}$ microscopy and connectomic techniques push for high resolution and large-scale imaging of brain tissues at microscopic resolutions, parallel advances in relating diffusion indices with more realistic models will likely follow. Importantly, the fidelity of the simulation geometry to the true underlying biology is a major concern. To the extent possible, these should encompass realistic and accurate distributions of axon diameters consistent with histological reports, random packing as opposed to a regular 
lattice, and other physical features that have been validated in tissue preparations. Although geometrical models are simplistic, simulations ensure that the estimated parameters are at least accurate and precise with respect to known ground truth features, and if this criteria is not met, acceptable performance in real tissues is likely compromised.

\section{Phantoms}

Ground truth hardware phantoms have been developed to estimate relevant parameters and calibrate multi-center studies. Ideally, phantoms mimicking WM structure possess certain desirable qualities most notably being tubular hollow structures with diameters approximating those of axons. Another desirable feature is to organize axon-like structures in different configurations that mimic complex features such as dispersed and crossing fibers. Intrinsic diffusivity of the filling material may also be considered, as well as magnetic susceptibility of the scaffolding material. Vegetables with tubular structures or domains, such as celery, asparagus, and chives, have been routinely used to demonstrate the effects of anisotropic diffusion, but these substances are clearly not amenable to long-term or cross-center studies, and phantoms with high consistency and longevity were sought. Most hardware phantoms have so far been developed for the purpose of optimizing DTI acquisitions or validating tractography rather than microstructure models. Textile fiber phantoms were developed with the scope of easily mimicking bending or dispersed fibers but their diameters typically far exceed the range of axons and they are of course not hollow [97]. Microcapillaries have the advantage of producing both an intra-axonal and an extra-axonal space to be filled [98-100], and most recent designs display somewhat more realistic diameters between 9 and 20 microns [101]. However, while crossing bundles can be designed, within each bundle the capillaries are highly aligned, which is a limitation for modeling true white matter.

Two types of phantoms have so far been developed for the purpose of validating higher order diffusion models. Fieremans et al. proposed a phantom of tightly packed solid fibers of Dyneema and compared the measured signal to simulations for diffusion, kurtosis and time dependence [102-104]. While it used reasonable diameters $(20 \mu \mathrm{m})$ the drawback was that it only modeled the extra-axonal space. Better magnetic susceptibility matching between water and the Dyneema material can be achieved using magnesium chloride [105]. A complementary phantom design of the intra-axonal space has recently been proposed using co-electrospun fibers that produce a hollow honeycomb-like arrangement and a distribution of diameters of about $9.5 \mu \mathrm{m}$ [106]. The ideal phantom for diffusion microstructure model validation would mimic both intra- and extra-axonal water with diameters matching those of white matter axons, randomly packed to appropriate volume fractions, and orientation dispersion. Clearly, it is challenging to meet all of these conditions simultaneously.

The advantages of phantoms are well recognized and aim to be standardized instruments for calibration of MRI experiments and assessments of multi-site variability. The disadvantages include sophisticated chemistry, limited availability to materials and specialized equipment, and non-standardization. Despite these current limitations, the role of consistent and widely-accepted phantoms has been well-recognized [107]. With the push for reproducibility and precision medicine, physical phantoms will continue to emerge as a solution to multi-center and multivendor diffusion MRI data acquisition and modeling.

\section{Validating Microstructural Features Fiber Orientation and Dispersion}

Diffusion MRI and its associated models offer the unique ability to track fiber pathways non-invasively. Diffusion tractography has been widely used in research settings to probe structural connectivity in health and disease and has seen success in clinical scenarios. Most notably, tractography has been used to identify fiber pathways during tumor resection [108] or other neurosurgical applications [109]. Tractography is based on the coherence in fiber orientation between adjacent voxels and is therefore fundamentally based on the accuracy of the estimated fiber orientation within each voxel. Validation efforts to demonstrate the accuracy of diffusion MRI have typically focused on applications to tractography, but these findings are also pertinent to microstructural modeling to derive local (i.e., voxel level) estimates of fiber orientations and dispersion. While the diffusion tensor models a single fiber orientation within each voxel, several methods have been developed to provide
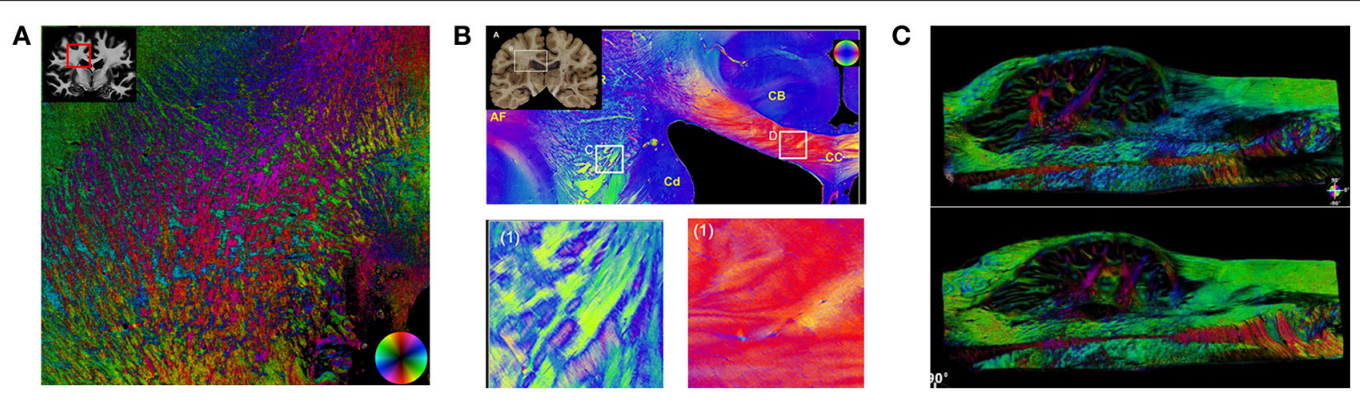

FIGURE 3 | Fiber Orientation Measurements in Fixed Tissues. Examples of fiber orientation measurements in human brain specimens. Structure tensor analysis (A) uses a digital texture analysis method to detect fiber orientations in stained sections imaged with bright-field or fluorescence microscopy. Both polarized light imaging (B) and polarization-sensitive optical coherence tomography (C) exploit the intrinsic birefringence of myelin to obtain fiber orientations. Sections in (A) [114] and (B) [115] (reproduced under the Creative Commons Attribution License) are from the human brain white matter, and (C) [116] from the human medulla oblongata (with permission). 
a comprehensive estimate of the number and distribution of fiber orientations within each voxel [64, 110], each with their own implications for tractography. Although, they have potential applications to tractography [111-113], diffusion MRI microstructural models aim to estimate intra-voxel orientation dispersion as its own unique feature to reveal interesting characteristics of the healthy, developing, or diseased brain.

Validating the intravoxel fiber dispersion, or ODF, is typically accomplished through imaging of the tissues using light microscopy or similar modalities and deriving quantities reflecting the neurite orientations (Figure 3). Thus, there is a more direct relationship between diffusion MRI quantities and those measured from histological samples than for volume fractions (see following section) because fiber orientations are largely unaffected by fixation and can be reliably measured in tissues with relative ease. Among the first examples to conduct direct validation studies, manual segmentation of axons in stained tissue white matter sections were compared to DTI and Diffusion Spectrum Imaging (DSI) estimates [117]. A variety of automated image processing techniques have been used to derive the angular orientation profiles of stained tissues, permitting large-scale automated analysis of tissue specimens [118-127]. These methods generally fall into classes of algorithms that use pattern-matching, Fourier analysis, or edge detection. Pattern matching attempts to identify discrete fibers within the image to build a full distribution of fiber dispersion. On the other hand, Fourier analysis and structure tensor analysis instead use the texture of the images to derive angular profiles of edges or spectral content. Structure tensor analysis has been particularly attractive since its interpretation is analogous to the diffusion tensor. Typically, the histological specimens were limited by twodimensional samples, but the structure tensor analysis has also been extended to 3D sections using confocal imaging [128, 129]. Collectively, the results from these studies all demonstrate a consistent and expected finding that while DTI fails to capture the full complexity of the underlying fiber orientation distributions, diffusion models that include dispersion as a feature better reflect the underlying structures. However, most studies thus far have validated orientation in tractography-driven approaches that focus solely on retrieving orientation without regard for diffusivities and volume fractions and do not fall into the category of microstructure models.

Stained histological sections for validation have several disadvantages and potential complications and confounds. Tissue processing and cutting can lead to distortion or artifacts, and histological staining may also be complicated by staining irregularity or artifacts. Moreover, sectioning, staining, and imaging large volumes at high magnification is very time consuming [130]. Although the brains and spinal cords of small animals can be captured with most laboratory microscopes, imaging large-scale tissues at high resolution such as human brain slices may require custom equipment and highperformance computing. Advances in large-scale microscopic imaging of fixed tissues have circumvented some of the issues with traditional sectioning and staining by using blockface imaging and intrinsic contrasts. Myelin exhibits the property of birefringence [131] which permits imaging of fiber architecture in fixed but unstained tissue sections. Somewhat analogous to diffusion encoding, rotating the polarization angle along different directions enables the estimation of fiber orientations in both $2 \mathrm{D}$ and $3 \mathrm{D}$ to examine human brain sections in their entirety [115, 132, 133]. Blockface microscopy, which consists of repeated imaging of the exposed surface of a tissue cut using serial sectioning, has also emerged as a powerful tool for large-scale imaging. Notably, compared to traditional sectioning, blockface imaging reduces distortions and the full process can be automated [116, 134, 135]. Modalities such as two-photon microscopy [136] or optical coherence tomography [137, 138] also image up to tens of microns below the surface and can provide contiguous 3D images of large tissue. Many of the whole-specimen imaging techniques developed for local neuron connectomic studies [139-141] have demonstrated utility when coupled with diffusion MRI [142].

Direct histological examination with microscopy has consistently demonstrated that white matter tracts are not strictly composed of uniform bundles. Even in the corpus callosum, an intravoxel dispersion of $\sim 18-20$ degrees is consistently evident from rodent [117], and human samples $[118,143]$. In the gray matter, Jespersen et al. demonstrated strong agreement between the estimated neurite orientation distributions and histological staining [144].

\section{Volume Fractions or Neurite Density}

The ability of diffusion MRI to monitor axon content in the brain and spinal cord has demonstrated wide ranging utility in a variety of applications across normal development, aging, injury, and disease. Unlike fiber orientation, which is generally preserved in fixed tissues, the relative volume fractions are perturbed during fixation and processing which has made nonMR measurements somewhat biased and inconsistent. It is important to first distinguish the true physical cellular fractions obtained from microscopy or other non-MRI methods from those reported from diffusion MRI models. In the brain, the extracellular volume fraction is typically around 20\% [145], but traditional tissue fixation and processing artificially reduces this to $\sim 2-5 \%$ [146]. In white matter, the axoplasm constitutes $\sim 25-30 \%$ of the physical space in white matter tissues [147], myelin constitutes $\sim 20-30 \%$ [147, 148], and glial cell bodies and processes constitute $\sim 30-40 \%$ [147]. Recent extracellular space-preserving methods of fixation support the approximation of 15-20\% extracellular space [149] and indicate that axonal volumes are relatively unaffected by the method of fixation [150]. In typical diffusion MRI experiments, the signal from water associated with myelin has a negligible contribution to the overall signal due to its short $T_{2}$ and is therefore routinely ignored in diffusion models. Diffusion MRI measures of compartment signal fractions are commonly referred to as neurite density or axonal volume fraction. Since density implies axonal counts and axonal volume fraction is ambiguous due to the myelin volume fraction, the term axonal water fraction (AWF) is a more precise terminology. Thus, derived from physical measurements alone, the AWF in healthy WM should be 33\% [147, 148]. However, to further complicate things, the relative fractions of the remaining 
"MR-visible" compartments are also weighted by their respective $T_{2}$ 's.

Several studies have compared MR-derived axon water fractions with direct histologic preparations of the same samples. Estimates of the intra-axonal volume fractions from DWI models consistently emerge around $40-60 \%[38,151]$, which is generally in line with histologic estimates although slightly higher than estimated from microscopy [152]. Jespersen et al. demonstrated agreement between neurite density estimates from DWI and quantitative microscopy, notably using both ex vivo DWI and histology [38, 61], and others have shown similarly strong relationships [153], although lower fractions have been noted in some samples [154]. Correlations (or absence thereof) between diffusion-derived and electron microscopy (EM)-derived metrics have also helped further establish the selective sensitivity of biomarkers derived from diffusion modeling to different features. For example, using WMTI, AWF has been shown to correlate with axon volume fraction but not with the $g$-ratio derived from EM, while $D_{\mathrm{e}, \perp}$ correlated with the $g$-ratio but not the with axon volume fraction [56]. Measures of AWF have also been combined with MRI measures of myelin content to estimate myelin gratio in vivo, which compared favorably to that derived from EM $[155,156]$. However, a strong correlation is not necessarily proof of parameter specificity. Notably, axon fractions derived from microscopy typically correlate very strongly with radial diffusivity $(\mathrm{RD})$ derived from the diffusion tensor [61].

\section{Compartment-Specific Diffusivities}

Diffusivities of the various modeled cellular compartments are both critical features that form the basis for diffusion models, and have been arguably the most difficult to validate. Alternative methods to NMR for measuring the self-diffusion coefficient of water are not available, and NMR-based measurements include signals from all compartments. In order to circumvent this issue and gain information about compartment-specific diffusivities, alternative approaches have been proposed: the use of model systems, endogenous compartment-specific metabolites, exogenous compartment-specific probes and tracers, signal suppression in a given compartment, exploiting alternative encoding methods, analyzing time-dependent properties, and combining diffusion methods with other contrasts $\left(T_{2}\right)$. Early studies examined compartment-specific diffusivities to glean insight into the nature of diffusion in the nervous system, with a particular focus on the biophysical basis of changes in cerebral ischemia. More recently, as highlighted in Part 1, a two-compartment model of diffusion shows a degeneracy that only an independent determination of compartment diffusivities can lift.

Model systems using invertebrates with large axons were first used to identify the source of anisotropy in the nervous system [157], demonstrating that biological membranes were the primary determinants of diffusion restriction/hindrance. These studies measured the intrinsic intra-axonal diffusivity, demonstrating it was $\sim 70-80 \%$ that of pure water $[158,159]$. Hence, these studies set the stage showing that intra-axonal diffusion was largely unrestricted along the axon, and the axonal membrane serves as a considerable barrier to diffusion perpendicular to the fibers.

Endogenous MRI-detectable tracers to assess diffusion primarily include metabolites detected through proton magnetic resonance spectroscopy (MRS). These include NAA, creatine, choline, and myo-inositol. NAA is a neuronal-specific metabolite and a surrogate marker of the intra-axonal space in white matter. Although, the neuronal specificity of NAA has recently been called into question since it was found to be also localized in myelin [160], the influence of myelin-associated NAA on the MR signal remains unclear and diffusion of NAA has so far been used to probe intra-axonal diffusion. Kroenke et al. [37] performed diffusion-weighted MRS in the human corpus callosum and rat brain, demonstrating that in vivo the $D_{\mathrm{a}, \|}$ of NAA was $\sim 50 \%\left(0.36 \mu \mathrm{m}^{2} / \mathrm{ms}\right)$ of that of an aqueous solution and $D_{\mathrm{a}, \perp}$ was effectively zero. Similar results were shown for NAA in the human brain and in a peripheral nerve preparation [161, 162]. Ronen et al. [143], accounting for both the macro- and microscopic curvature of the human corpus callosum, measured a slightly larger $D_{\mathrm{a}, \|}\left(0.51 \mu \mathrm{m}^{2} / \mathrm{ms}\right)$ putting it in the range of $60-70 \%$ of aqueous NAA. Palombo et al. [163] have also demonstrated diffusion of NAA and other metabolites can be modeled as occurring in long, cylindrical fibers, having a $D_{\mathrm{a}, \|}$ of $0.33 \mu \mathrm{m}^{2} / \mathrm{ms}$. The diffusion weighted signals of other metabolites were also fit well by assuming long cylindrical processes [163-165]. Since metabolites have differences in molecular size, affinity to charged surfaces, and potential ambiguity in compartment selectivity, the intra-axonal diffusivity of water cannot be unequivocally extrapolated from metabolite diffusivities. However, these studies and those in experimental preparations are consistent with the intra-axonal water diffusivity being $60-80 \%$ of its temperature-matched aqueous diffusivity.

Exogenous MRI-detectable tracers, most notably injectable agents used in animal models, have also been used to probe the diffusivity of specific environments. Non-proton probes have been used to selectively probe either the extracellular space (sodium-based [166]), the intracellular space (cesiumbased [167]) or each of them separately (fluorine-based [168]). The diffusivity of extracellular proton-based agents such as mannitol, phenylphosphonate, and polyethylene glycols has also been investigated using diffusion spectroscopy [169]. Often though, the compartment specificity is not perfect and transmembrane exchange is a complicating factor [170]. Nonetheless, using these approaches, it has been suggested that the diffusivities in the intra- and extracellular environments for molecules of these sizes are approximately similar to one another. It should be noted though that these measurements were typically limited to the apparent diffusion coefficient (ADC) in rat gray matter, and should rather be interpreted as: the traces of the diffusion tensors in the intra- and extracellular environments are approximately similar to one another.

Compartment selectivity can be also achieved by suppressing the extracellular water signal using exogenous tracers. Intracerebroventricular infusion of a gadolinium-based contrast agent causes the $T_{2}$ of the extracellular space to decrease, 
effectively suppressing its contribution during signal acquisition. Following measurements in the rat gray matter, Silva et al. reported similar ADCs with and without the suppression, which supports the idea of comparable diffusion traces in the intraand extracellular environments for water molecules as well [171]. Recently, this experiment was replicated in the rat corpus callosum in a direction-specific manner and concluded that $D_{\mathrm{a}, \|}$ $\geq D_{\mathrm{e}, \|}[172]$.

Non contrast-based alternatives using non-conventional diffusion encoding have also provided insight into how axial compartment diffusivities compare. A double diffusion encoding (DDE) sequence was recently used to exploit $D_{\mathrm{a}, \perp}=0$ and suppress the extra-axonal signal using a strong initial diffusion gradient perpendicular to the spinal cord, with the results suggesting $D_{\mathrm{a}, \|} \approx D_{\mathrm{e}, \|}$ [173]. Isotropic diffusion weighting has been used by two independent groups to show that isotropic kurtosis is negligible in most brain structures, including white matter tracts [174, 175], whereby the traces of the intraand extra-axonal compartments were similar. This recurrent observation implied the $D_{\mathrm{a}, \|} \geq D_{\mathrm{e}, \|}$ solution of the twocompartment model was valid. Most recently, Jespersen et al. have shown also that the time-dependence for compartmentspecific diffusivities in a Watson-WMTI model is physically acceptable only for $D_{\mathrm{a}, \|} \geq D_{\mathrm{e}, \|}$, albeit in fixed rat spinal cord [59]. The inclusion of compartment $\mathrm{T}_{2}$ 's in the LEMONADE model also suggested likewise [176]. Thus, evidence from a variety of experiments has generally favored $D_{\mathrm{a}, \|} \geq D_{\mathrm{e}, \|}$, although true independent validation is still lacking.

\section{Validation of Model Parameter Choices}

The choice and accuracy of diffusion model parameters can be gleaned from these validation studies. The first observation is that, for clinical diffusion times, the long-time limit applies and intra-axonal diffusion perpendicular to a single fiber $\left(D_{\mathrm{a}, \perp}\right)$ is effectively zero: thus the "stick" model of axons is generally appropriate for axon diameters in the central nervous system, as has also been demonstrated recently [177]. Second, under this stick model assumption, the orientations of fibers examined histologically are reasonably well-approximated by diffusion models that account for dispersion or crossing-fibers, but it should be noted that most of these studies have not jointly considered dispersion along with simultaneous estimates of other microstructural properties. Third, the intra-axonal parallel diffusivity $\left(D_{\mathrm{a}, \|}\right)$ is $\sim 60-80 \%$ of the temperature-matched aqueous solution of the same molecule $\left(D_{\text {free }}\right)$. Fourth, in the healthy white matter, the intra-axonal parallel diffusivity $D_{\mathrm{a}, \|}$ is likely faster than the extra-axonal diffusivity $D_{\mathrm{e}, \|}$, although not substantially.

\section{PARAMETER CHOICE IN THE INJURED AND DISEASED WHITE MATTER}

In the healthy white matter, diffusion models have converged to a relatively uniform set of parameters as described previously, which has been proposed as the universal model of white matter diffusion. To avoid model overfitting, each of the biophysical model variants neglects, constrains, or holds fixed certain parameters while leaving others free to fit to the data. Importantly, while these constraints have nuanced effects in the healthy brain and spinal cord, there is still considerable disparity and disagreement about the necessary and relevant model parameters in the injured or diseased brain. A complication arises in which the pathologies of different injuries or diseases may be different from one another and are likely to evolve over time and therefore, since there is no consistent pathology, there is unlikely to be a diffusion model that universally captures it. Moreover, while a specific pathology can lead to predictable diffusion MRI outcomes, many different pathologies can lead to the same diffusion behavior. Furthermore, single pathologies rarely occur in isolation. While the diffusion model parameters themselves are independent, there are often strong correlations between pairs of diffusion parameters and specific pathological features.

Diffusion MRI has been applied to nearly every brain and spinal cord disease or injury. DTI represents the overwhelming majority of studies related to validation, yet many investigators have conducted comprehensive studies to relate biophysical model parameters with the gold-standard techniques. While a comprehensive review of all of the different disease and injury categories is beyond the scope here, highlighting specific examples will shed light on the issues.

\section{Cerebral Ischemia}

The most common clinically useful application of diffusion MRI is in the detection of acute cerebral ischemia [178, 179]. Within minutes of the onset, diffusion within the infarct is decreased by approximately one half. Although the precise mechanism has been debated, it is closely related to the loss of membrane polarization, ion imbalance, and cell swelling characteristic of acute ischemia [180]. Compartment-specific tracer studies have indicated that the intracellular and extracellular compartments exhibit similar decreases in diffusion $[168,171]$, while others have shown that intracellular diffusivity decreases substantially while the extracellular diffusivity experiences a somewhat lesser decrease [167]. The extracellular decrease is consistent with a reduction in the extracellular space and increase in tortuosity that leads to decreased diffusivity in that compartment [33], while the intracellular decrease is consistent with neurite beading $[94,181]$. Oscillating gradient measurements in the rat brain have demonstrated that restrictions caused by ischemia occur on the scale of several microns typical of cell sizes [182]. The reduced diffusion in stroke can also relate to a larger fraction of highly restricted diffusion $[183,184]$ which may be compatible with a still water compartment $\left(D_{\text {iso }}=0\right)$. Clearly, as the injured tissue degenerates, multiple coincident pathologies exist and the interpretation of diffusion MRI changes becomes increasingly more complex. For example, both NODDI and WMTI have been applied to acute human stroke, with contrasting results. WMTI demonstrated a large reduction in $D_{\mathrm{a}, \|}$ in coherent fiber pathways, with lesser reductions in $D_{\mathrm{e}, \|}$ (under the assumption $\left.D_{\mathrm{a}, \|}<D_{\mathrm{e}, \|}\right)$ consistent with neurite beading hypothesis [13]. On the other hand, NODDI applied to human stroke demonstrated 
pronounced increases in the estimated orientation dispersion [42]. Although these differences may be related to the time after onset, animal models reveal almost no changes to neurite organization in the acute aftermath [185]. Thus, the model assumptions of NODDI of fixed diffusivities appear to cause misleading interpretations when those assumptions are strongly violated.

\section{Demyelination and Myelin Disorders}

Demyelination and other myelin disorders, despite having hallmark pathologies of myelin loss, degeneration, or dysfunction, also typically exhibit multiple overlapping pathologies. Particularly in the acute inflammatory phases of the disease or model, myelin pathologies are accompanied by microglia and astrocyte activation and proliferation [186], and axonal injury is also prominent in some models of multiple sclerosis or optic neuritis [187]. Thus, multiple confounding pathologies present significant challenges of both specificity and sensitivity in relation to diffusion models. A chemical model of demyelination induced through cuprizone toxicity has been used by many groups to validate diffusion and other MRI markers related to specific pathologies. Using the WMTI framework, Guglielmetti et al. [12] demonstrated a prominent reduction in $D_{\mathrm{a}, \|}$ during the acute inflammatory phase, while a reduced AWF was observed throughout the remyelination and recovery period. Jelescu et al. [56] showed that the axonal fraction assessed with electron microscopy correlated with AWF derived from WMTI, while the g-ratio correlated with $D_{e, \perp}$. In a model of hypomyelination, Kelm et al. [58] demonstrated strong correlations between axonal fractions obtained from histology and MRI, again using WMTI, but no significant correlations between other WMTI parameters and histology measures were found. Using the DBSI framework, Wang et al. [67] demonstrated strong correlations between axial diffusivity (a DBSI parameter related to $D_{\mathrm{a}, \|}$ ) and axon integrity, radial diffusivity (a parameter related to $D_{\mathrm{e}, \perp}$ ), and myelinated axons, and cell ratio (a parameter related to $D_{\text {iso }}$ ) and histological cell counts, with similar findings reported in the experimental autoimmune encephalomyelitis [72] and optic neuritis [68] animal models. However, most disease models examined previously with DTI $[188,189]$ demonstrated strong associations between many of the microscopy measures and quantitative diffusion MRI measures, thus strong correlations are not an indicator of specificity. These studies highlight the challenges of diffusion MRI-based biomarkers since many different pathologies can give rise to similar diffusion signatures and separate pathologies rarely occur in isolation from one another. Moreover, it is worth reiterating that diffusion is an indirect marker of the entire water content of the voxel, and while high specificity is desired, the reliability, sensitivity, and clinical usefulness of these techniques will also be paramount.

\section{SUMMARY}

Diffusion MRI is uniquely sensitive to microscopic tissue features that are nearly impossible to achieve through other means. However, diffusion MRI is intrinsically limited since it is an indirect measure of tissue microstructure and relies on inferences from models and estimation of relevant parameters. A plethora of approaches have emerged. The biologicallyinspired models have many similarities although each has different inherent assumptions and algorithms. Consequently, validating diffusion models and derived parameters is necessary to demonstrate accuracy, and has relied on various approaches. Simulations and phantoms have a role in demonstrating the accuracy and precision to a known ground truth and can identify sources of error and effects of violating the underlying assumptions. The predominant approach to validate diffusion MRI models is optical imaging of fixed tissue specimens since it offers a direct assessment of the physical features of the tissues and their true biological complexity. An increasing body of literature has demonstrated that diffusion models which account for intra-voxel fiber dispersion are generally good approximations to the underlying white matter fiber organization. Estimates of axonal volume fraction with diffusion MRI are also approximately consistent with histological measurements when accounting for potential bias of fixation and tissue processing. The intrinsic diffusivities of the intra- and extra-axonal compartments are more challenging to validate independently since they can only be measured using NMR itself, and alternative diffusion encodings seem promising in providing the sought validation. In the injured and diseased white matter, considerable ambiguity still exists in the choice of model parameters along with their imposed constraints. Critically, the range of possible pathologies is likely to be too complex to be accurately captured with a single universal model and avoid over-fitting. Validation studies will be important to establish disease and pathology specific applications. Ultimately, the goal of diffusion MRI modeling is to provide a clinically meaningful insight for diagnosis and treatment efficacy.

\section{AUTHOR CONTRIBUTIONS}

All authors listed have made a substantial, direct, and intellectual contribution to the work, and approved it for publication.

\section{FUNDING}

IJ acknowledges support from the Centre d'Imagerie Biomédicale (CIBM) of the University of Lausanne (UNIL), the Swiss Federal Institute of Technology Lausanne (EPFL), the University of Geneva (UniGe), the Centre Hospitalier Universitaire Vaudois (CHUV), the Hôpitaux Universitaires de Genève (HUG) and the Leenaards and the Jeantet Foundations. MDB acknowledges support from the Craig $H$. Neilsen Foundation (297024), the US Department of Veterans Affairs Rehabilitation Research and Development Service (Merit Review Award I01 RX001497), and the Bryon Riesch Paralysis Foundation. 


\section{REFERENCES}

1. Novikov DS, Jespersen SN, Kiselev VG, Fieremans E. Quantifying brain microstructure with diffusion MRI: Theory and parameter estimation. arXiv:1612.02059 [physics.bio-ph] (2016a).

2. Jensen JH, Helpern JA, Ramani A, Lu H, Kaczynski K. Diffusional kurtosis imaging: the quantification of non-gaussian water diffusion by means of magnetic resonance imaging. Magn Reson Med. (2005) 53:1432-40. doi: $10.1002 / \mathrm{mrm} .20508$

3. Kiselev VG. The cumulant expansion: an overarching mathematical framework for understanding diffusion NMR. In: Jones, DK, editor. Diffusion MRI: Theory, Methods, and Applications. Oxford: Oxford University Press (2010), p. 152-68.

4. Van Kampen NG. Stochastic Processes in Physics and Chemistry. 3rd edn. North Holland, North Holland (2007).

5. Basser PJ, Mattiello J, LeBihan D. Estimation of the effective self-diffusion tensor from the NMR spin echo. J Magn Reson B. (1994) 103:247-54. doi: 10.1006/jmrb.1994.1037

6. Jones DK. Precision and accuracy in diffusion tensor magnetic resonance imaging. Top Magn Reson Imaging (2010) 21:87-99. doi: 10.1097/RMR.0b013e31821e56ac

7. Veraart J, Sijbers J, Sunaert S, Leemans A, Jeurissen B. Weighted linear least squares estimation of diffusion MRI parameters: strengths, limitations, and pitfalls. Neuroimage (2013) 81:335-46. doi: 10.1016/j.neuroimage.2013.05.028

8. Veraart J, Poot DH, Van Hecke W, Blockx I, Van der Linden A, Verhoye M, et al. More accurate estimation of diffusion tensor parameters using diffusion Kurtosis imaging. Magn Reson Med. (2011) 65:138-45. doi: $10.1002 / \mathrm{mrm} .22603$

9. Kiselev VG. Fundamentals of diffusion MRI physics. NMR Biomed. (2017) 30:e3602. doi: $10.1002 / \mathrm{nbm} .3602$

10. Mori S (editor). Chapter 11: Application studies. In: Introduction to Diffusion Tensor Imaging. Amsterdam: Elsevier Science B.V (2007), p. 149-61.

11. Van Hecke W, Emsell L, Sunaert S. Diffusion Tensor Imaging - A Practical Handbook. 1st ed. New York, NY: Springer-Verlag (2016).

12. Guglielmetti C, Veraart J, Roelant E, Mai Z, Daans J, Van Audekerke $\mathrm{J}$, et al. Diffusion kurtosis imaging probes cortical alterations and white matter pathology following cuprizone induced demyelination and spontaneous remyelination. Neuroimage (2016) 125:363-77. doi: 10.1016/j.neuroimage.2015.10.052

13. Hui ES, Fieremans E, Jensen JH, Tabesh A, Feng W, Bonilha L, et al. Stroke assessment with diffusional kurtosis imaging. Stroke (2012) 43:2968-73. doi: 10.1161/STROKEAHA.112.657742

14. Jensen JH, Helpern JA. MRI quantification of non-Gaussian water diffusion by kurtosis analysis. NMR Biomed. (2010) 23:698-710. doi: $10.1002 / \mathrm{nbm} .1518$

15. Kochunov P, Rowland LM, Fieremans E, Veraart J, Jahanshad N, Eskandar $\mathrm{G}$, et al. Diffusion-weighted imaging uncovers likely sources of processingspeed deficits in schizophrenia. Proc Natl Acad Sci USA. (2016) 113:13504-9. doi: $10.1073 /$ pnas. 1608246113

16. Paydar A, Fieremans E, Nwankwo JI, Lazar M, Sheth HD, Adisetiyo V, et al. Diffusional kurtosis imaging of the developing brain. AJNR Am J Neuroradiol. (2014) 35:808-14. doi: 10.3174/ajnr.A3764

17. Rosenkrantz AB, Padhani AR, Chenevert TL, Koh DM, De Keyzer F, Taouli $\mathrm{B}$, et al. Body diffusion kurtosis imaging: basic principles, applications, and considerations for clinical practice. J Magn Reson Imaging (2015) 42:11901202. doi: $10.1002 /$ jmri.24985

18. Hansen B, Lund TE, Sangill R, Jespersen SN. Experimentally and computationally fast method for estimation of a mean kurtosis. Magn Reson Med. (2013) 69:1754-60. doi: 10.1002/mrm.24743

19. Hansen B, Shemesh N, Jespersen SN. Fast imaging of mean, axial and radial diffusion kurtosis. Neuroimage (2016) 142:381-93. doi: 10.1016/j.neuroimage.2016.08.022

20. Yablonskiy DA, Bretthorst GL, Ackerman JJ. Statistical model for diffusion attenuated MR signal. Magn Reson Med. (2003) 50, 664-9. doi: $10.1002 / \mathrm{mrm} .10578$

21. Grinberg F, Farrher E, Ciobanu L, Geffroy F, Le Bihan D, Shah NJ. Non-Gaussian diffusion imaging for enhanced contrast of brain tissue affected by ischemic stroke. PLoS ONE (2014) 9:e89225. doi: 10.1371 /journal.pone. 0089225
22. Novikov DS, Kiselev VG. Effective medium theory of a diffusion-weighted signal. NMR Biomed. (2010) 23:682-97. doi: 10.1002/nbm.1584

23. Bennett KM, Schmainda KM, Bennett RT, Rowe DB, Lu H, Hyde JS. Characterization of continuously distributed cortical water diffusion rates with a stretched-exponential model. Magn Reson Med. (2003) 50:727-34. doi: $10.1002 / \mathrm{mrm} .10581$

24. Ingo C, Magin RL, Colon-Perez L, Triplett W, Mareci TH. On random walks and entropy in diffusion-weighted magnetic resonance imaging studies of neural tissue. Magn Reson Med. (2014) 71:617-27. doi: 10.1002/mrm.24706

25. Palombo M, Gabrielli A, De Santis S, Cametti C, Ruocco G, Capuani S. Spatio-temporal anomalous diffusion in heterogeneous media by nuclear magnetic resonance. J Chem Phys. (2011) 135:034504. doi: $10.1063 / 1.3610367$

26. Zhou XJ, Gao Q, Abdullah O, Magin RL. Studies of anomalous diffusion in the human brain using fractional order calculus. Magn Reson Med. (2010) 63:562-9. doi: $10.1002 / \mathrm{mrm} .22285$

27. Bai Y, Lin Y, Tian J, Shi D, Cheng J, Haacke EM, et al. Grading of gliomas by using monoexponential, biexponential, and stretched exponential diffusionweighted MR imaging and diffusion kurtosis MR imaging. Radiology (2016) 278:496-504. doi: 10.1148/radiol.2015142173

28. Lai V, Lee VH, Lam KO, Sze HC, Chan Q, Khong PL. Intravoxel water diffusion heterogeneity MR imaging of nasopharyngeal carcinoma using stretched exponential diffusion model. Eur Radiol. (2015) 25:1708-13. doi: 10.1007/s00330-014-3535-9

29. Liu X, Zhou L, Peng W, Wang H, Zhang Y. Comparison of stretchedExponential and monoexponential model diffusion-Weighted imaging in prostate cancer and normal tissues. J Magn Reson Imaging (2015) 42:107885. doi: $10.1002 / j m r i .24872$

30. Mazaheri Y, Afaq A, Rowe DB, Lu Y, Shukla-Dave A, Grover J. Diffusionweighted magnetic resonance imaging of the prostate: improved robustness with stretched exponential modeling. J Comput Assist Tomogr. (2012) 36:695-703. doi: 10.1097/RCT.0b013e31826bdbbd

31. Özarslan E, Koay CG, Shepherd TM, Komlosh ME, Irfanoglu MO, Pierpaoli $\mathrm{C}$, et al. Mean apparent propagator (MAP) MRI: a novel diffusion imaging method for mapping tissue microstructure. Neuroimage (2013) 78:16-32. doi: 10.1016/j.neuroimage.2013.04.016

32. Avram AV, Sarlls JE, Barnett AS, Ozarslan E, Thomas C, Irfanoglu MO, et al. Clinical feasibility of using mean apparent propagator (MAP) MRI to characterize brain tissue microstructure. Neuroimage (2016) 127:422-34. doi: 10.1016/j.neuroimage.2015.11.027

33. Latour LL, Svoboda K, Mitra PP, Sotak CH. Time-dependent diffusion of water in a biological model system. Proc Natl Acad Sci USA. (1994) 91:1229-33. doi: 10.1073/pnas.91.4.1229

34. Szafer A, Zhong J, Gore JC. Theoretical model for water diffusion in tissues. Magn Reson Med. (1995) 33:697-712. doi: 10.1002/mrm.1910330516

35. Stanisz GJ, Szafer A, Wright GA, Henkelman RM. An analytical model of restricted diffusion in bovine optic nerve. Magn Reson Med. (1997) 37:103-11. doi: 10.1002/mrm.1910370115

36. Behrens TE, Woolrich MW, Jenkinson M, Johansen-Berg H, Nunes RG, Clare S, et al. Characterization and propagation of uncertainty in diffusion-weighted MR imaging. Magn Reson Med. (2003) 50:1077-88. doi: $10.1002 / \mathrm{mrm} .10609$

37. Kroenke CD, Ackerman JJ, Yablonskiy DA. On the nature of the NAA diffusion attenuated MR signal in the central nervous system. Magn Reson Med. (2004) 52:1052-59. doi: 10.1002/mrm.20260

38. Jespersen SN, Kroenke CD, Ostergaard L, Ackerman JJ, Yablonskiy DA. Modeling dendrite density from magnetic resonance diffusion measurements. Neuroimage (2007) 34:1473-86. doi: 10.1016/j.neuroimage.2006.10.037

39. Mikula S, Denk W. High-resolution whole-brain staining for electron microscopic circuit reconstruction. Nat Methods (2015) 12:541-6. doi: 10.1038/nmeth.3361

40. Lampinen B, Szczepankiewicz F, van Westen D, Englund E, Sundgren PC, Lätt $\mathrm{J}$, et al. Optimal experimental design for filter exchange imaging: apparent exchange rate measurements in the healthy brain and in intracranial tumors. Magn Reson Med. (2017) 77:1104-14. doi: $10.1002 / \mathrm{mrm} .26195$

41. Zhang $\mathrm{H}$, Schneider $\mathrm{T}$, Wheeler-Kingshott CA, Alexander DC NODDI: practical in vivo neurite orientation dispersion and density imaging of the human brain. Neuroimage (2012) 61:1000-16. doi: 10.1016/j.neuroimage.2012.03.072 
42. Adluru G, Gur Y, Anderson JS, Richards LG, Adluru N, DiBella EV. Assessment of white matter microstructure in stroke patients using NODDI. Conf Proc IEEE Eng Med Biol Soc. (2014) 2014:742-5. doi: 10.1109/EMBC.2014.6943697

43. Churchill NW, Caverzasi E, Graham SJ, Hutchison MG, Schweizer TA. White matter microstructure in athletes with a history of concussion: comparing diffusion tensor imaging (DTI) and neurite orientation dispersion and density imaging (NODDI). Hum Brain Mapp. (2017) 38:4201-11. doi: 10.1002/hbm.23658

44. Kunz N, Zhang H, Vasung L, O’Brien KR, Assaf Y, Lazeyras F, et al. Assessing white matter microstructure of the newborn with multi-shell diffusion MRI and biophysical compartment models. Neuroimage (2014) 96, 288-99. doi: 10.1016/j.neuroimage.2014.03.057

45. Okita G, Ohba T, Takamura T, Ebata S, Ueda R, Onishi H, et al. Application of neurite orientation dispersion and density imaging or diffusion tensor imaging to quantify the severity of cervical spondylotic myelopathy and assess postoperative neurological recovery. Spine J. (2017). doi: 10.1016/j.spinee.2017.07.007. [Epub ahead of print].

46. Schneider T, Brownlee W, Zhang H, Ciccarelli O, Miller DH, WheelerKingshott CG. Sensitivity of multi-shell NODDI to multiple sclerosis white matter changes: a pilot study. Funct Neurol. (2017) 32:97-101. doi: 10.11138/FNeur/2017.32.2.097

47. Slattery CF, Zhang J, Paterson RW, Foulkes AJM, Carton, A, Macpherson, $\mathrm{K}$, et al. ApoE influences regional white-matter axonal density loss in Alzheimer's disease. Neurobiol Aging (2017) 57:8-17. doi: 10.1016/j.neurobiolaging.2017.04.021

48. Wen Q, Kelley DA, Banerjee S, Lupo JM, Chang SM, Xu D, et al. Clinically feasible NODDI characterization of glioma using multiband EPI at $7 \mathrm{~T}$. Neuroimage Clin. (2015) 9:291-9. doi: 10.1016/j.nicl.2015.08.017

49. Jelescu IO, Veraart J, Fieremans E, Novikov DS. Degeneracy in model parameter estimation for multi-compartmental diffusion in neuronal tissue. NMR Biomed. (2016a) 29, 33-47. doi: 10.1002/nbm.3450

50. Novikov DS, Veraart J, Jelescu IO, Fieremans E. Mapping orientational and microstructural metrics of neuronal integrity with in vivo diffusion MRI. arXiv:1609.09144 (2016b).

51. Novikov DS, Fieremans E. Relating extracellular diffusivity to cell size distribution and packing density as applied to white matter. In: Proc Intl Soc Mag Reson Med. Melbourne, VIC (2012). p. 1829.

52. Fieremans E, Jensen JH, Helpern JA. White matter characterization with diffusional kurtosis imaging. Neuroimage (2011) 58:177-88. doi: 10.1016/j.neuroimage.2011.06.006

53. Fieremans E, Novikov DS, Jensen JH, Helpern JA. Monte Carlo study of a two-compartment exchange model of diffusion. NMR Biomed. (2010) 23:711-24. doi: 10.1002/nbm.1577

54. Benitez A, Fieremans E, Jensen JH, Falangola MF, Tabesh A, Ferris SH, et al. White matter tract integrity metrics reflect the vulnerability of latemyelinating tracts in Alzheimer's disease. Neuroimage Clin. (2013) 4:64-71. doi: 10.1016/j.nicl.2013.11.001

55. de Kouchkovsky I, Fieremans E, Fleysher L, Herbert J, Grossman RI, Inglese M. Quantification of normal-appearing white matter tract integrity in multiple sclerosis: a diffusion kurtosis imaging study. J Neurol. (2016) 263:1146-55, doi: 10.1007/s00415-016-8118-Z

56. Jelescu IO, Zurek M, Winters KV, Veraart J, Rajaratnam A, Kim NS, et al. In vivo quantification of demyelination and recovery using compartmentspecific diffusion MRI metrics validated by electron microscopy. Neuroimage (2016b) 132:104-14. doi: 10.1016/j.neuroimage.2016.02.004

57. Kamiya K, Hori M, Irie R, Miyajima M, Nakajima M, Kamagata K, et al. Diffusion imaging of reversible and irreversible microstructural changes within the corticospinal tract in idiopathic normal pressure hydrocephalus. Neuroimage Clin. (2017) 14:663-71. doi: 10.1016/j.nicl.2017.03.003

58. Kelm ND, West KL, Carson RP, Gochberg DF, Ess KC, Does MD. Evaluation of diffusion kurtosis imaging in ex vivo hypomyelinated mouse brains. Neuroimage (2016) 124:612-26. doi: 10.1016/j.neuroimage.2015.09.028

59. Jespersen SN, Olesen JL, Hansen B, Shemesh N. Diffusion time dependence of microstructural parameters in fixed spinal cord. Neuroimage (2017). doi: 10.1016/j.neuroimage.2017.08.039. [Epub ahead of print].

60. Tariq M, Schneider T, Alexander DC, Gandini Wheeler-Kingshott CA, Zhang H. Bingham-NODDI: mapping anisotropic orientation dispersion of neurites using diffusion MRI. Neuroimage (2016) 133, 207-23. doi: 10.1016/j.neuroimage.2016.01.046

61. Jespersen SN, Bjarkam CR, Nyengaard JR, Chakravarty MM, Hansen B, Vosegaard T, et al. Neurite density from magnetic resonance diffusion measurements at ultrahigh field: comparison with light microscopy and electron microscopy. Neuroimage (2010) 49:205-16. doi: 10.1016/j.neuroimage.2009.08.053

62. Anderson AW. Measurement of fiber orientation distributions using high angular resolution diffusion imaging. Magn Reson Med. (2005) 54:1194-206. doi: 10.1002/mrm.20667

63. Dell'Acqua F, Rizzo G, Scifo P, Clarke RA, Scotti G, Fazio F. A model-based deconvolution approach to solve fiber crossing in diffusionweighted MR imaging. IEEE Trans Biomed Eng. (2007) 54:462-72. doi: 10.1109/TBME.2006.888830

64. Tournier JD, Calamante F, Gadian DG, Connelly A. Direct estimation of the fiber orientation density function from diffusion-weighted MRI data using spherical deconvolution. Neuroimage (2004) 23:1176-85. doi: 10.1016/j.neuroimage.2004.07.037

65. Reisert M, Kellner E, Dhital B, Hennig J, Kiselev VG. Disentangling micro from mesostructure by diffusion MRI: a Bayesian approach. Neuroimage (2017) 147:964-75. doi: 10.1016/j.neuroimage.2016.09.058

66. Kaden E, Kelm ND, Carson RP, Does MD, Alexander DC. Multicompartment microscopic diffusion imaging. Neuroimage (2016) 139:346-59. doi: 10.1016/j.neuroimage.2016.06.002

67. Wang Y, Wang Q, Haldar JP, Yeh FC, Xie M, Sun P, et al. Quantification of increased cellularity during inflammatory demyelination. Brain (2011) 134:3590-601. doi: 10.1093/brain/awr307

68. Chiang CW, Wang Y, Sun P, Lin TH, Trinkaus K, Cross AH, et al. Quantifying white matter tract diffusion parameters in the presence of increased extra-fiber cellularity and vasogenic edema. Neuroimage (2014) 101:310-9. doi: 10.1016/j.neuroimage.2014.06.064

69. Lin TH, Chiang CW, Perez-Torres CJ, Sun P, Wallendorf M, Schmidt RE, et al. Diffusion MRI quantifies early axonal loss in the presence of nerve swelling. J Neuroinflammation (2017) 14:78. doi: 10.1186/s12974-017-0852-3

70. Murphy RK, Sun P, Xu J, Wang Y, Sullivan S, Gamble P, et al. Magnetic resonance imaging biomarker of axon loss reflects cervical spondylotic myelopathy severity. Spine (2016) 41:751-6. doi: 10.1097/BRS.0000000000001337

71. Sun P, Murphy RK, Gamble P, George A, Song SK, Ray WZ. Diffusion assessment of cortical changes, induced by traumatic spinal cord injury. Brain Sci. (2017) 7:E21. doi: 10.3390/brainsci7020021

72. Wang X, Cusick MF, Wang Y, Sun P, Libbey JE, Trinkaus K, et al. Diffusion basis spectrum imaging detects and distinguishes coexisting subclinical inflammation, demyelination and axonal injury in experimental autoimmune encephalomyelitis mice. NMR Biomed. (2014c) 27:843-52. doi: $10.1002 / \mathrm{nbm} .3129$

73. Pierpaoli C, Jones DK. Removing CSF contamination in brain DT-MRIs by using a two-compartment tensor model. In: Proc Intl Soc Mag Reson Med. Kyoto (2004). p. 1215

74. Pasternak O, Sochen N, Gur Y, Intrator N, Assaf Y. Free water elimination and mapping from diffusion MRI. Magn Reson Med. (2009) 62:717-30. doi: $10.1002 / \mathrm{mrm} .22055$

75. Albi A, Pasternak O, Minati L, Marizzoni M, Bartres-Faz D, Bargallo N, et al. Free water elimination improves test-retest reproducibility of diffusion tensor imaging indices in the brain: a longitudinal multisite study of healthy elderly subjects. Hum Brain Mapp. (2017) 38:12-26. doi: 10.1002/hbm.23350

76. Bergamino M, Pasternak O, Farmer M, Shenton ME, Hamilton JP. Applying a free-water correction to diffusion imaging data uncovers stressrelated neural pathology in depression. Neuroimage Clin. (2016) 10:336-42. doi: 10.1016/j.nicl.2015.11.020

77. Oestreich LK, Pasternak O, Shenton ME, Kubicki M, Gong X, McCarthyJones S, et al. Abnormal white matter microstructure and increased extracellular free-water in the cingulum bundle associated with delusions in chronic schizophrenia. Neuroimage Clin. (2016) 12:405-14. doi: 10.1016/j.nicl.2016.08.004

78. Ofori E, Pasternak O, Planetta PJ, Li H, Burciu RG, Snyder AF, et al. Longitudinal changes in free-water within the substantia nigra of Parkinson's disease. Brain (2015) 138:2322-31. doi: 10.1093/brain/awv136 
79. Pasternak O, Shenton ME, Westin CF. Estimation of extracellular volume from regularized multi-shell diffusion MRI. Med Image Comput Comp Assist Interv. (2012) 15:305-12. doi: 10.1007/978-3-642-33418-4_38

80. Hoy AR, Koay CG, Kecskemeti SR, Alexander AL. Optimization of a free water elimination two-compartment model for diffusion tensor imaging. Neuroimage (2014) 103:323-33. doi: 10.1016/j.neuroimage.2014.09.053

81. Chou MC, Lin YR, Huang TY, Wang CY, Chung HW, Juan CJ, et al. FLAIR diffusion-tensor MR tractography: comparison of fiber tracking with conventional imaging. AJNR Am J Neuroradiol. (2005) 26:591-7.

82. Hoy AR, Kecskemeti SR, Alexander AL. Free water elimination diffusion tractography: a comparison with conventional and fluid-attenuated inversion recovery, diffusion tensor imaging acquisitions. J Magn Reson Imaging (2015) 42:1572-81. doi: 10.1002/jmri.24925

83. Scherrer B, Schwartzman A, Taquet M, Sahin M, Prabhu SP, Warfield SK. Characterizing brain tissue by assessment of the distribution of anisotropic microstructural environments in diffusion-compartment imaging (DIAMOND). Magn Reson Med. (2016) 76:963-77. doi: 10.1002/mrm.25912

84. Ferizi U, Schneider T, Panagiotaki E, Nedjati-Gilani G, Zhang H, WheelerKingshott CA, et al. A ranking of diffusion MRI compartment models with in vivo human brain data. Magn Reson Med. (2014) 72:1785-92. doi: $10.1002 / \mathrm{mrm} .25080$

85. Panagiotaki E, Schneider T, Siow B, Hall MG, Lythgoe MF, Alexander DC. Compartment models of the diffusion MR signal in brain white matter: a taxonomy and comparison. Neuroimage (2012) 59, 2241-54. doi: 10.1016/j.neuroimage.2011.09.081

86. Scherrer B, Qiu J, Hashim J, Afacan O, Wu Y, Marcotrigiano M, et al. Diffusion compartment imaging reveals microstructural injuries in a mouse model of mild traumatic brain injury. In: Proc Intl Soc Mag Reson Med. Honolulu, HI (2017). p. 286.

87. Jelescu IO, Veraart J, Adisetiyo V, Milla SS, Novikov DS, Fieremans E. One diffusion acquisition and different white matter models: how does microstructure change in human early development based on WMTI and NODDI? Neuroimage (2015) 107:242-56. doi: 10.1016/j.neuroimage.2014.12.009

88. Wang S, Peterson DJ, Wang Y, Wang Q, Grabowski TJ, Li W, et al. Empirical comparison of diffusion kurtosis imaging and diffusion basis spectrum imaging using the same acquisition in healthy young adults. Front Neurol. (2017) 8:118. doi: 10.3389/fneur.2017.00118

89. Balls GT, Frank LR. A simulation environment for diffusion weighted MR experiments in complex media. Magn Reson Med. (2009) 62, 771-8. doi: $10.1002 / \mathrm{mrm} .22033$

90. Hall MG, Alexander DC. Convergence and parameter choice for MonteCarlo simulations of diffusion MRI. IEEE Trans Med Imaging (2009) 28:1354-64. doi: 10.1109/TMI.2009.2015756

91. Mingasson T, Duval T, Stikov N, Cohen-Adad J. AxonPacking: an opensource software to simulate arrangements of axons in white matter. Front. Neuroinform. (2017) 11:5. doi: 10.3389/fninf.2017.00005

92. Yeh CH, Schmitt B, Le Bihan D, Li-Schlittgen JR, Lin CP, Poupon C. Diffusion microscopist simulator: a general Monte Carlo simulation system for diffusion magnetic resonance imaging. PLoS ONE (2013) 8:e76626. doi: 10.1371/journal.pone.0076626

93. Landman BA, Farrell JA, Smith SA, Reich DS, Calabresi PA, van Zijl PC. Complex geometric models of diffusion and relaxation in healthy and damaged white matter. NMR Biomed. (2010) 23:152-62. doi: $10.1002 /$ nbm.1437

94. Budde MD, Frank JA. Neurite beading is sufficient to decrease the apparent diffusion coefficient after ischemic stroke. Proc Natl Acad Sci USA. (2010) 107:14472-7. doi: 10.1073/pnas.1004841107

95. Nilsson M, Latt J, Stahlberg F, van Westen D, Hagslatt H. The importance of axonal undulation in diffusion MR measurements: a Monte Carlo simulation study. NMR Biomed. (2012) 25:795-805. doi: 10.1002/nbm. 1795

96. Panagiotaki E, Hall MG, Zhang H, Siow B, Lythgoe MF, Alexander DC. High-fidelity meshes from tissue samples for diffusion MRI simulations. Med. Image Comput. Comp. Assist. Interv. (2010) 13(Pt 2):404-11. doi: 10.1007/978-3-642-15745-5_50

97. Perrin M, Poupon C, Rieul B, Leroux P, Constantinesco A, Mangin JF, et al. Validation of q-ball imaging with a diffusion fibre-crossing phantom on a clinical scanner. Philos Trans R Soc Lond B Biol Sci. (2005) 360:881-91. doi: $10.1098 /$ rstb.2005.1650
98. Kim SJ, Choi CG, Kim JK, Yun SC, Jahng GH, Jeong HK, et al. Effects of MR parameter changes on the quantification of diffusion anisotropy and apparent diffusion coefficient in diffusion tensor imaging: evaluation using a diffusional anisotropic phantom. Korean J Radiol. (2015) 16:297-303. doi: 10.3348/kjr.2015.16.2.297

99. Oida T, Nagahara S, Kobayashi T. Acquisition parameters for diffusion tensor imaging to emphasize fractional anisotropy: phantom study. Magn Reson Med Sci. (2011) 10:121-128. doi: 10.2463/mrms.10.121

100. Tournier JD, Yeh CH, Calamante F, Cho KH, Connelly A, Lin CP. Resolving crossing fibres using constrained spherical deconvolution: validation using diffusion-weighted imaging phantom data. Neuroimage (2008) 42:617-25. doi: 10.1016/j.neuroimage.2008.05.002

101. Komlosh ME, Benjamini D, Barnett AS, Schram V, Horkay F, Avram AV, et al. Anisotropic phantom to calibrate high-q diffusion MRI methods. J Magn Reson. (2017) 275:19-28. doi: 10.1016/j.jmr.2016.11.017

102. Burcaw LM, Fieremans E, Novikov DS. Mesoscopic structure of neuronal tracts from time-dependent diffusion. Neuroimage (2015) 114:18-37. doi: 10.1016/j.neuroimage.2015.03.061

103. Fieremans E, De Deene Y, Delputte S, Ozdemir MS, Achten E, Lemahieu I. The design of anisotropic diffusion phantoms for the validation of diffusion weighted magnetic resonance imaging. Phys Med Biol. (2008a) 53:5405-19. doi: 10.1088/0031-9155/53/19/009

104. Fieremans E, De Deene Y, Delputte S, Özdemir MS, D'Asseler Y, Vlassenbroeck J, et al. Simulation and experimental verification of the diffusion in an anisotropic fiber phantom. J. Magn Reson. (2008b) 190:189-99. doi: 10.1016/j.jmr.2007.10.014

105. Farrher E, Lindemeyer J, Grinberg F, Oros-Peusquens A.-M, Shah, NJ. Concerning the matching of magnetic susceptibility differences for the compensation of background gradients in anisotropic diffusion fibre phantoms. PLOS ONE (2017) 12:e0176192. doi: 10.1371/journal.pone.0176192

106. Hubbard PL, Zhou FL, Eichhorn SJ, Parker GJ. Biomimetic phantom for the validation of diffusion magnetic resonance imaging. Magn Reson Med (2015) 73:299-305. doi: 10.1002/mrm.25107

107. Palacios EM, Martin AJ, Boss MA, Ezekiel F, Chang YS, Yuh EL, et al. Toward precision and reproducibility of diffusion tensor imaging: a multicenter diffusion phantom and traveling volunteer study. AJNR Am J Neuroradiol. (2017) 38:537-45. doi: 10.3174/ajnr.A5025

108. Nimsky C, Ganslandt O, Hastreiter P, Wang R, Benner T, Sorensen AG, et al. Preoperative and intraoperative diffusion tensor imaging-based fiber tracking in glioma surgery. Neurosurgery (2005) 56:130-7; discussion: 138. doi: 10.1227/01.NEU.0000144842.18771.30

109. Johansen-Berg H, Behrens TE. Just pretty pictures? What diffusion tractography can add in clinical neuroscience. Curr Opin Neurol. (2006) 19:379-385. doi: 10.1097/01.wco.0000236618.82086.01

110. Tuch DS, Reese TG, Wiegell MR, Wedeen VJ. Diffusion MRI of complex neural architecture. Neuron (2003) 40:885-95. doi: 10.1016/S0896-6273(03)00758-X

111. Christiaens D, Reisert M, Dhollander T, Sunaert S, Suetens P, Maes F. Global tractography of multi-shell diffusion-weighted imaging data using a multi-tissue model. Neuroimage (2015) 123:89-101. doi: 10.1016/j.neuroimage.2015.08.008

112. Daducci A, Dal Palu A, Descoteaux M, Thiran JP. Microstructure informed tractography: pitfalls and open challenges. Front Neurosci (2016) 10:247. doi: 10.3389/fnins.2016.00247

113. Reisert M, Kiselev VG, Dihtal B, Kellner E, Novikov DS. MesoFT: unifying diffusion modelling and fiber tracking. Med Image Comput Comp Assist Interv. (2014) 17:201-8. doi: 10.1007/978-3-319-10443-0_26

114. Budde MD, Annese J. Quantification of anisotropy and fiber orientation in human brain histological sections. Front Integr Neurosci. (2013a) 7:3. doi: 10.3389/fnint.2013.00003

115. Axer M, Strohmer S, Gräßel D, Bücker O, Dohmen M, Reckfort J, et al. Estimating fiber orientation distribution functions in 3D-Polarized Light Imaging. Front. Neuroanat. (2016) 10:40. doi: 10.3389/fnana.2016.00040

116. Wang H, Zhu J, Reuter M, Vinke LN, Yendiki A, Boas DA, et al. Crossvalidation of serial optical coherence scanning and diffusion tensor imaging: a study on neural fiber maps in human medulla oblongata. Neuroimage (2014b) 100:395-404. doi: 10.1016/j.neuroimage.2014.06.032

117. Leergaard TB, White NS, de Crespigny A, Bolstad I, D'Arceuil H, Bjaalie JG, et al. Quantitative histological validation of diffusion MRI fiber 
orientation distributions in the rat brain. PLoS ONE (2010) 5:e8595. doi: 10.1371/journal.pone.0008595

118. Budde MD, Annese J. Quantifying anisotropy and fiber orientation in human brain histological sections. Front Integr Neurosci. (2013b) 7:3. doi: $10.3389 /$ fnint.2013.00003

119. Budde MD, Frank JA. Examining brain microstructure using structure tensor analysis of histological sections. Neuroimage (2012) 63:1-10. doi: 10.1016/j.neuroimage.2012.06.042

120. Choe AS, Stepniewska I, Colvin DC, Ding Z, Anderson AW. Validation of diffusion tensor MRI in the central nervous system using light microscopy: quantitative comparison of fiber properties. NMR Biomed. (2012) 25:900-8. doi: $10.1002 / \mathrm{nbm} .1810$

121. Gangolli M, Holleran L, Hee Kim J, Stein TD, Alvarez V, McKee AC, et al. Quantitative validation of a nonlinear histology-MRI coregistration method using generalized Q-sampling imaging in complex human cortical white matter. Neuroimage (2017) 153:152-67. doi: 10.1016/j.neuroimage.2017.03.059

122. Grussu F, Schneider T, Yates RL, Zhang H, Wheeler-Kingshott CAMG, DeLuca GC, et al. A framework for optimal whole-sample histological quantification of neurite orientation dispersion in the human spinal cord. J. Neurosci. Methods (2016) 273:20-32. doi: 10.1016/j.jneumeth.2016.08.002

123. Hansen B, Flint JJ, Heon-Lee C, Fey M, Vincent F, King MA, et al. Diffusion tensor microscopy in human nervous tissue with quantitative correlation based on direct histological comparison. Neuroimage (2011) 57:1458-65. doi: 10.1016/j.neuroimage.2011.04.052

124. Mitter C, Jakab A, Brugger PC, Ricken G, Gruber GM, Bettelheim D, et al. Validation of in utero tractography of human fetal commissural and internal capsule fibers with histological structure tensor analysis. Front. Neuroanat. (2015) 9:164. doi: 10.3389/fnana.2015.00164

125. Mollink J, Kleinnijenhuis M, Cappellen van Walsum AMV, Sotiropoulos SN, Cottaar M, Mirfin C, et al. Evaluating fibre orientation dispersion in white matter: comparison of diffusion MRI, histology and polarized light imaging. Neuroimage (2017) 157:561-74. doi: 10.1016/j.neuroimage.2017.06.001

126. Nazaran A, Wisco JJ, Hageman N, Schettler SP, Wong A, Vinters HV, et al. Methodology for computing white matter nerve fiber orientation in human histological slices. J Neurosci Methods (2016) 261:75-84. doi: 10.1016/j.jneumeth.2015.11.023

127. Seehaus A, Roebroeck A, Bastiani M, Fonseca L, Bratzke H, Lori N, et al. Histological validation of high-resolution DTI in human post mortem tissue. Front Neuroanat. (2015) 9:98. doi: 10.3389/fnana.2015.00098

128. Khan AR, Cornea A, Leigland LA, Kohama SG, Jespersen SN, Kroenke CD. 3D structure tensor analysis of light microscopy data for validating diffusion MRI. Neuroimage (2015) 111:192-203. doi: 10.1016/j.neuroimage.2015.01.061

129. Schilling K, Janve V, Gao Y, Stepniewska I, Landman BA, Anderson AW. Comparison of 3D orientation distribution functions measured with confocal microscopy and diffusion MRI. Neuroimage (2016) 129:185-97. doi: 10.1016/j.neuroimage.2016.01.022

130. Annese J. The importance of combining MRI and large-scale digital histology in neuroimaging studies of brain connectivity and disease. Front Neuroinform. (2012) 6:13. doi: 10.3389/fninf.2012.00013

131. de Campos Vidal B, Mello ML, Caseiro-Filho AC, Godo C. Anisotropic properties of the myelin sheath. Acta Histochem. (1980) 66:32-9. doi: $10.1016 /$ S0065-1281(80)80079-1

132. Axer H, Beck S, Axer M, Schuchardt F, Heepe J, Flucken A, et al. Microstructural analysis of human white matter architecture using polarized light imaging: Views from neuroanatomy. Front Neuroinform. (2011a) 5:28. doi: $10.3389 /$ fninf.2011.00028

133. Axer M, Amunts K, Grässel D, Palm C, Dammers J, Axer H, et al. A novel approach to the human connectome: ultra-high resolution mapping of fiber tracts in the brain. Neuroimage (2011b) 54:1091-101. doi: 10.1016/j.neuroimage.2010.08.075

134. Wang H, Lenglet C, Akkin T. Structure tensor analysis of serial optical coherence scanner images for mapping fiber orientations and tractography in the brain. J Biomed Optics (2015) 20:036003. doi: 10.1117/1.JBO.20.3. 036003

135. Wang H, Zhu J, Akkin T. Serial optical coherence scanner for large-scale brain imaging at microscopic resolution. Neuroimage (2014a) 84:1007-1017. doi: 10.1016/j.neuroimage.2013.09.063
136. Oh SW, Harris JA, Ng L, Winslow B, Cain N, Mihalas S, et al. A mesoscale connectome of the mouse brain. Nature (2014) 508:207-14. doi: 10.1038/nature13186

137. Magnain C, Augustinack JC, Reuter M, Wachinger C, Frosch MP, Ragan $\mathrm{T}$, et al. Blockface histology with optical coherence tomography: a comparison with Nissl staining. Neuroimage (2014) 84:524-33. doi: 10.1016/j.neuroimage.2013.08.072

138. Srinivasan VJ, Radhakrishnan H, Jiang JY, Barry S, Cable AE. Optical coherence microscopy for deep tissue imaging of the cerebral cortex with intrinsic contrast. Opt Express (2012) 20:2220-39. doi: 10.1364/OE.20.002220

139. Denk W, Horstmann H. Serial block-face scanning electron microscopy to reconstruct three-dimensional tissue nanostructure. PLoS Biol. (2004) 2:e329. doi: 10.1371/journal.pbio.0020329

140. Dodt HU, Leischner U, Schierloh A, Jährling N, Mauch CP, Deininger $\mathrm{K}$, et al. Ultramicroscopy: three-dimensional visualization of neuronal networks in the whole mouse brain. Nat Methods (2007) 4:331-6. doi: 10.1038/nmeth1036

141. Mikula S, Binding J, Denk W. Staining and embedding the whole mouse brain for electron microscopy. Nat Methods (2012) 9:1198-201. doi: $10.1038 /$ nmeth.2213

142. Chang EH, Argyelan M, Aggarwal M, Chandon T. SS, Karlsgodt KH, Mori S, et al. The role of myelination in measures of white matter integrity: combination of diffusion tensor imaging and two-photon microscopy of CLARITY intact brains. Neuroimage (2017) 147:119-24. doi: 10.1016/j.neuroimage.2016.11.068

143. Ronen I, Budde M, Ercan E, Annese J, Techawiboonwong A, Webb A. Microstructural organization of axons in the human corpus callosum quantified by diffusion-weighted magnetic resonance spectroscopy of $\mathrm{N}$ acetylaspartate and post-mortem histology. Brain Struct Funct. (2014) 219:1773-85. doi: 10.1007/s00429-013-0600-0

144. Jespersen SN, Leigland LA, Cornea A, Kroenke CD. Determination of axonal and dendritic orientation distributions within the developing cerebral cortex by diffusion tensor imaging. IEEE Trans Med Imaging (2012) 31:16-32. doi: 10.1109/TMI.2011.2162099

145. Sykova E, Nicholson C. Diffusion in brain extracellular space. Physiol Rev. (2008) 88:1277-340. doi: 10.1152/physrev.00027.2007

146. Cragg B. Preservation of extracellular space during fixation of the brain for electron microscopy. Tissue Cell (1980) 12:63-72. doi: 10.1016/0040-8166(80)90052-X

147. Perge JA, Koch K, Miller R, Sterling P, Balasubramanian V. How the optic nerve allocates space, energy capacity, and information. J Neurosci. (2009) 29:7917-28. doi: 10.1523/JNEUROSCI.5200-08.2009

148. Ong HH, Wright AC, Wehrli SL, Souza A, Schwartz ED, Hwang SN, et al. Indirect measurement of regional axon diameter in excised mouse spinal cord with q-space imaging: simulation and experimental studies. Neuroimage (2008) 40:1619-32. doi: 10.1016/j.neuroimage.2008.01.017

149. Pallotto M, Watkins PV, Fubara B, Singer JH, Briggman KL. Extracellular space preservation aids the connectomic analysis of neural circuits. Elife (2015) 4:e08206. doi: 10.7554/eLife.08206

150. Korogod N, Petersen CC, Knott GW. Ultrastructural analysis of adult mouse neocortex comparing aldehyde perfusion with cryo fixation. Elife (2015) 4:e05793. doi: 10.7554/eLife.05793

151. Grussu F, Schneider T, Zhang H, Alexander DC, Wheeler-Kingshott CA. Neurite orientation dispersion and density imaging of the healthy cervical spinal cord in vivo. Neuroimage (2015) 111:590-601. doi: 10.1016/j.neuroimage.2015.01.045

152. Chklovskii DB, Schikorski T, Stevens CF. Wiring optimization in cortical circuits. Neuron (2002) 34:341-7. doi: 10.1016/S0896-6273(02)00679-7

153. Sepehrband F, Clark KA, Ullmann JF, Kurniawan ND, Leanage G, Reutens DC, et al. Brain tissue compartment density estimated using diffusionweighted MRI yields tissue parameters consistent with histology. Hum Brain Mapp. (2015) 36:3687-702. doi: 10.1002/hbm.22872

154. Golabchi FN, Brooks DH, Hoge WS, De Girolami U, Maier SE. Pixelbased comparison of spinal cord MR diffusion anisotropy with axon packing parameters. Magn Reson Med. (2010) 63:1510-9. doi: 10.1002/mrm.22337

155. Duval T, Le Vy S, Stikov N, Campbell J, Mezer A, Witzel T, et al. g-Ratio weighted imaging of the human spinal cord in vivo. Neuroimage (2017) 145:11-23. doi: 10.1016/j.neuroimage.2016.09.018 
156. Stikov N, Campbell JS, Stroh T, Lavelee M, Frey S, Novek J, et al. In vivo histology of the myelin g-ratio with magnetic resonance imaging. Neuroimage (2015) 118:397-405. doi: 10.1016/j.neuroimage.2015.05.023

157. Beaulieu C. The basis of anisotropic water diffusion in the nervous system a technical review. NMR Biomed. (2002) 15:435-55. doi: 10.1002/nbm.782

158. Takahashi M, Hackney DB, Zhang G, Wehrli SL, Wright AC, O'Brien WT, et al. Magnetic resonance microimaging of intraaxonal water diffusion in live excised lamprey spinal cord. Proc Natl Acad Sci USA. (2002) 99:16192-6. doi: 10.1073/pnas.252249999

159. Beaulieu C, Allen PS. Water diffusion in the giant axon of the squid: implications for diffusion-weighted MRI of the nervous system. Magn Reson Med. (1994) 32:579-83. doi: 10.1002/mrm.1910320506

160. Nordengen K, Heuser C, Rinholm JE, Matalon R, Gundersen V. Localisation of N-acetylaspartate in oligodendrocytes/myelin. Brain Struct Funct. (2015) 220:899-917. doi: 10.1007/s00429-013-0691-7

161. Ellegood J, Hanstock CC, Beaulieu C. Diffusion tensor spectroscopy (DTS) of human brain. Magn Reson Med. (2006) 55:1-8. doi: 10.1002/mrm.20777

162. Ellegood J, McKay RT, Hanstock CC, Beaulieu C. Anisotropic diffusion of metabolites in peripheral nerve using diffusion weighted magnetic resonance spectroscopy at ultra-high field. J Magn Reson. (2007) 184:20-28. doi: 10.1016/j.jmr.2006.09.008

163. Palombo M, Ligneul C, Valette J. Modeling diffusion of intracellular metabolites in the mouse brain up to very high diffusion-weighting: diffusion in long fibers (almost) accounts for non-monoexponential attenuation. Magn Reson Med. (2017) 77:343-50. doi: 10.1002/mrm.26548

164. Najac C, Branzoli F, Ronen I, Valette J. Brain intracellular metabolites are freely diffusing along cell fibers in grey and white matter, as measured by diffusion-weighted MR spectroscopy in the human brain at 7 T. Brain Struct Funct. (2016) 221:1245-1254. doi: 10.1007/s00429-014-0968-5

165. Najac C, Marchadour C, Guillermier M, Houitte D, Slavov V, Brouillet $\mathrm{E}$, et al. Intracellular metabolites in the primate brain are primarily localized in long fibers rather than in cell bodies, as shown by diffusionweighted magnetic resonance spectroscopy. Neuroimage (2014) 90:374-380. doi: 10.1016/j.neuroimage.2013.12.045

166. Goodman JA, Kroenke CD, Bretthorst GL, Ackerman JJ, Neil JJ. Sodium ion apparent diffusion coefficient in living rat brain. Magn Reson Med. (2005) 53:1040-1045. doi: 10.1002/mrm.20444

167. Goodman JA, Ackerman JJ, Neil JJ. Cs $+\mathrm{ADC}$ in rat brain decreases markedly at death. Magn Reson Med. (2008) 59:65-72. doi: $10.1002 / \mathrm{mrm} .21418$

168. Duong TQ, Ackerman JJ, Ying HS, Neil JJ. Evaluation of extra- and intracellular apparent diffusion in normal and globally ischemic rat brain via 19F NMR. Magn Reson Med. (1998) 40:1-13. doi: 10.1002/mrm.1910400102

169. Duong TQ, Sehy JV, Yablonskiy DA, Snider BJ, Ackerman JJ, Neil JJ. Extracellular apparent diffusion in rat brain. Magn Reson Med. (2001) 45:801-10. doi: 10.1002/mrm.1108

170. Ackerman JJ, Neil JJ. The use of MR-detectable reporter molecules and ions to evaluate diffusion in normal and ischemic brain. NMR Biomed. (2010) 23:725-33. doi: 10.1002/nbm.1530

171. Silva MD, Omae T, Helmer KG, Li F, Fisher M, Sotak CH. Separating changes in the intra- and extracellular water apparent diffusion coefficient following focal cerebral ischemia in the rat brain. Magn Reson Med. (2002) 48:826-37. doi: $10.1002 / \mathrm{mrm} .10296$

172. Jelescu IO, Kunz N, Da Silva AR, Gruetter R. Intra- and extra-axonal axial diffusivities in the white matter: which one is faster? In: Proc. Intl. Soc. Mag. Reson Med. Honolulu, HI (2017). p. 281.

173. Skinner NP, Kurpad SN, Schmit BD, Tugan Muftuler L, Budde MD. Rapid in vivo detection of rat spinal cord injury with double-diffusion-encoded magnetic resonance spectroscopy. Magn Reson Med. (2017) 77:1639-49. doi: $10.1002 / \mathrm{mrm} .26243$

174. Dhital B, Kellner E, Reisert M, Kiselev VG. Isotropic diffusion weighting provides insight on diffusion compartments in human brain white matter in vivo. In: Proc Intl Soc Mag Reson Med. Toronto, ON (2015). p. 2788.

175. Szczepankiewicz F, Lasic S, van Westen D, Sundgren PC, Englund E, Westin $\mathrm{CF}$, et al. Quantification of microscopic diffusion anisotropy disentangles effects of orientation dispersion from microstructure: applications in healthy volunteers and in brain tumors. Neuroimage (2015) 104:241-52. doi: 10.1016/j.neuroimage.2014.09.057
176. Veraart J, Novikov DS, Fieremans E. TE dependent Diffusion Imaging (TEdDI) distinguishes between compartmental T2 relaxation times. Neuroimage (2017). doi: 10.1016/j.neuroimage.2017.09.030. [Epub ahead of print].

177. Veraart J, Fieremans E, Novikov DS. Universal power-law scaling of water diffusion in human brain defines what we see with MRI. arXiv:1609.09145 [physics.bio-ph] (2016).

178. Le Bihan D, Johansen-Berg H. Diffusion MRI at 25: exploring brain tissue structure and function. Neuroimage (2012) 61:324-41. doi: 10.1016/j.neuroimage.2011.11.006

179. Moseley ME, Cohen Y, Mintorovitch J, Chileuitt L, Shimizu H, Kucharczyk J, et al. Early detection of regional cerebral ischemia in cats: comparison of diffusion- and T2-weighted MRI and spectroscopy. Magn Reson Med. (1990) 14:330-46. doi: 10.1002/mrm.1910140218

180. Benveniste H, Hedlund LW, Johnson GA. Mechanism of detection of acute cerebral ischemia in rats by diffusion-weighted magnetic resonance microscopy. Stroke (1992) 23:746-54. doi: 10.1161/01.STR. 23.5.746

181. Baron CA, Kate M, Gioia L, Butcher K, Emery D, Budde M, et al. Reduction of diffusion-weighted imaging contrast of acute ischemic stroke at short diffusion times. Stroke (2015) 46:2136-41. doi: 10.1161/STROKEAHA.115.008815

182. Does MD, Parsons EC, Gore JC. Oscillating gradient measurements of water diffusion in normal and globally ischemic rat brain. Magn Reson Med. (2003) 49:206-215. doi: 10.1002/mrm.10385

183. Brugieres P, Thomas P, Maraval A, Hosseini H, Combes C, Chafiq A, et al. Water diffusion compartmentation at high b values in ischemic human brain. AJNR Am J Neuroradiol. (2004) 25, 692-8.

184. Niendorf T, Dijkhuizen RM, Norris DG, van Lookeren Campagne M, Nicolay K. Biexponential diffusion attenuation in various states of brain tissue: implications for diffusion-weighted imaging. Magn Reson Med. (1996) 36:847-57. doi: 10.1002/mrm.1910360607

185. Li P, Murphy TH. Two-photon imaging during prolonged middle cerebral artery occlusion in mice reveals recovery of dendritic structure after reperfusion. J Neurosci. (2008) 28:11970-9. doi: 10.1523/JNEUROSCI.3724-08.2008

186. Lucchinetti C, Bruck W, Parisi J, Scheithauer B, Rodriguez M, Lassmann H. Heterogeneity of multiple sclerosis lesions: implications for the pathogenesis of demyelination. Ann Neurol. (2000) 47:707-17. doi: 10.1002/15318249(200006)47:6<707::AID-ANA3>3.0.CO;2-Q

187. Trapp BD, Peterson J, Ransohoff RM, Rudick R, Mork S, Bo L. Axonal transection in the lesions of multiple sclerosis. N Engl J Med. (1998) 338:278-85. doi: 10.1056/NEJM199801293380502

188. Budde MD, Xie M, Cross AH, Song SK. Axial diffusivity is the primary correlate of axonal injury in the experimental autoimmune encephalomyelitis spinal cord: a quantitative pixelwise analysis. $J$ Neurosci. (2009) 29:2805-13. doi: 10.1523/JNEUROSCI.4605-08. 2009

189. Thiessen JD, Zhang Y, Zhang H, Wang L, Buist R, Del Bigio MR, et al. Quantitative MRI and ultrastructural examination of the cuprizone mouse model of demyelination. NMR Biomed. (2013) 26:1562-81. doi: $10.1002 / \mathrm{nbm} .2992$

Conflict of Interest Statement: IJ is a co-inventor on U.S. Patent Application No. 15/156250, pending. "System, method and computer-accessible medium for determining brain microstructure parameters from diffusion magnetic resonance."

The other author declares that the research was conducted in the absence of any commercial or financial relationships that could be construed as a potential conflict of interest.

Copyright (c) 2017 Jelescu and Budde. This is an open-access article distributed under the terms of the Creative Commons Attribution License (CC BY). The use, distribution or reproduction in other forums is permitted, provided the original author(s) or licensor are credited and that the original publication in this journal is cited, in accordance with accepted academic practice. No use, distribution or reproduction is permitted which does not comply with these terms. 